TRANSACTIONS OF THE

AMERICAN MATHEMATICAL SOCIETY

Volume 360, Number 6, June 2008, Pages 3041-3069

S 0002-9947(08)04288-8

Article electronically published on January 8, 2008

\title{
EHRHART POLYNOMIALS OF LATTICE-FACE POLYTOPES
}

\author{
FU LIU
}

\begin{abstract}
There is a simple formula for the Ehrhart polynomial of a cyclic polytope. The purpose of this paper is to show that the same formula holds for a more general class of polytopes, lattice-face polytopes. We develop a way of decomposing any $d$-dimensional simplex in general position into $d$ ! signed sets, each of which corresponds to a permutation in the symmetric group $\mathfrak{S}_{d}$, and reduce the problem of counting lattice points in a polytope in general position to that of counting lattice points in these special signed sets. Applying this decomposition to a lattice-face simplex, we obtain signed sets with special properties that allow us to count the number of lattice points inside them. We are thus able to conclude the desired formula for the Ehrhart polynomials of lattice-face polytopes.
\end{abstract}

\section{INTRODUCTION}

A $d$-dimensional lattice $\mathbb{Z}^{d}=\left\{\mathbf{x}=\left(x_{1}, \ldots, x_{d}\right) \mid \forall x_{i} \in \mathbb{Z}\right\}$ is the collection of all points with integer coordinates in $\mathbb{R}^{d}$. Any point in a lattice is called a lattice point.

A convex polytope is a convex hull of a finite set of points. We often omit convex and just say polytope. For any polytope $P$ and some positive integer $m \in \mathbb{N}$, we use $i(m, P)$ to denote the number of lattice points in $m P$, where $m P=\{m x \mid x \in P\}$ is the $m$ th dilated polytope of $P$.

An integral or lattice polytope is a convex polytope whose vertices are all lattice points. Eugène Ehrhart [4] showed that for any $d$-dimensional integral polytope, $i(P, m)$ is a polynomial in $m$ of degree $d$. Thus, we call $i(P, m)$ the Ehrhart polynomial of $P$ when $P$ is an integral polytope. Please see $[2,3]$ for more references to the literature of lattice point counting. Although Ehrhart's theory was developed in the 1960's, we still do not know much about the coefficients of Ehrhart polynomials for general polytopes except that the leading, second and last coefficients of $i(P, m)$ are the normalized volume of $P$, one half of the normalized volume of the boundary of $P$ and 1 , respectively.

The moment curve in $\mathbb{R}^{d}$ is defined by $\nu_{d}: \mathbb{R} \rightarrow \mathbb{R}^{d}, t \mapsto \nu_{d}(t)=\left(t, t^{2}, \ldots, t^{d}\right)$. Let $T=\left\{t_{1}, \ldots, t_{n}\right\}$ be an $n$-element set. The cyclic polytope $C_{d}(T)=C_{d}\left(t_{1}, \ldots, t_{n}\right)$ is the convex hull conv $\left\{v_{d}\left(t_{1}\right), v_{d}\left(t_{2}\right), \ldots, v_{d}\left(t_{n}\right)\right\}$ of $n>d$ distinct points $\nu_{d}\left(t_{i}\right), 1 \leq$ $i \leq n$, on the moment curve. In [5], the author showed that for any $d$-dimensional

Received by the editors February 15, 2006 and, in revised form, March 15, 2006. 2000 Mathematics Subject Classification. Primary 05A19; Secondary 52B20.

Key words and phrases. Ehrhart polynomial, lattice-face, polytope, signed decomposition. 
cyclic polytope $P=C_{d}(T)$, we have that

$$
i(P, m)=\operatorname{Vol}(m P)+i(\pi(P), m)=\sum_{k=0}^{d} \operatorname{Vol}_{k}\left(\pi^{(d-k)}(P)\right) m^{k},
$$

where $\pi^{(k)}: \mathbb{R}^{d} \rightarrow \mathbb{R}^{d-k}$ is the map which ignores the last $k$ coordinates of a point, and asked whether there are other integral polytopes that have the the same form of Ehrhart polynomials.

In this paper, we define a new family of integral polytopes, lattice-face polytopes, and show (Theorem 3.5) that their Ehrhart polynomials are in the same form as (1.1).

The main method of [5] is a decomposition of an arbitrary $d$-dimensional simplex cyclic polytope into $d$ ! signed sets, each of which corresponds to a permutation in the symmetric group $\mathfrak{S}_{d}$ and has the same sign as the corresponding permutation. However, for general polytopes, such a decomposition does not work.

In this paper, we develop a way of decomposing any $d$-dimensional simplex in general position into $d$ ! signed sets, where the sign of each set is not necessarily the same as the corresponding permutation. Applying the new decomposition to a lattice-face simplex, we are able to show (Theorem 3.6) that the number of lattice points is given by a formula (6.1) involving Bernoulli polynomials, signs of permutations, and determinants, and then to analyze this formula further to derive the theorem. Theorem 3.6, together with some simple observations in section 2 and 3, implies Theorem 3.5.

\section{Preliminaries}

We first give some definitions and notation, most of which follows [5].

All polytopes we will consider are full-dimensional, so for any convex polytope $P$, we use $d$ to denote both the dimension of the ambient space $\mathbb{R}^{d}$ and the dimension of $P$. We call a $d$-dimensional polytope a $d$-polytope. Also, we use $\partial P$ and $I(P)$ to denote the boundary and the interior of $P$, respectively.

For any set $S$, we use $\operatorname{conv}(S)$ to denote the convex hull of all of points in $S$.

Recall that the projection $\pi: \mathbb{R}^{d} \rightarrow \mathbb{R}^{d-1}$ is the map that forgets the last coordinate. For any set $S \subset \mathbb{R}^{d}$ and any point $y \in \mathbb{R}^{d-1}$, let $\rho(y, S)=\pi^{-1}(y) \cap S$ be the intersection of $S$ with the inverse image of $y$ under $\pi$. Let $p(y, S)$ and $n(y, S)$ be the point in $\rho(y, S)$ with the largest and smallest last coordinate, respectively. If $\rho(y, S)$ is the empty set, i.e., $y \notin \pi(S)$, then let $p(y, S)$ and $n(y, S)$ be empty sets as well. Clearly, if $S$ is a $d$-polytope, $p(y, S)$ and $n(y, S)$ are on the boundary of $S$. Also, we let $\rho^{+}(y, S)=\rho(y, S) \backslash n(y, S)$, and for any $T \subset \mathbb{R}^{d-1}, \rho^{+}(T, S)=\bigcup_{y \in T} \rho^{+}(y, S)$.

Definition 2.1. Define $P B(P)=\bigcup_{y \in \pi(P)} p(y, P)$ to be the positive boundary of $P ; N B(P)=\bigcup_{y \in \pi(P)} n(y, P)$ to be the negative boundary of $P$ and $\Omega(P)=$ $P \backslash N B(P)=\rho^{+}(\pi(P), P)=\bigcup_{y \in \pi(P)} \rho^{+}(y, P)$ to be the nonnegative part of $P$.

Definition 2.2. For any facet $F$ of $P$, if $F$ has an interior point in the positive boundary of $P$, then we call $F$ a positive facet of $P$ and define the sign of $F$ as $+1: \operatorname{sign}(F)=+1$. Similarly, we can define the negative facets of $P$ with associated sign -1 . For the facets that are neither positive nor negative, we call them neutral facets and define the sign as 0. 
It's easy to see that $F \subset P B(P)$ if $F$ is a positive facet and $F \subset N B(P)$ if $F$ is a negative facet.

We write $P=\bigsqcup_{i=1}^{k} P_{i}$ if $P=\bigcup_{i=1}^{k} P_{i}$ and for any $i \neq j, P_{i} \cap P_{j}$ is contained in their boundaries. If $F_{1}, F_{2}, \ldots, F_{\ell}$ are all the positive facets of $P$ and $F_{\ell+1}, \ldots, F_{k}$ are all the negative facets of $P$, then

$$
\pi(P)=\bigsqcup_{i=1}^{\ell} \pi\left(F_{i}\right)=\bigsqcup_{i=\ell+1}^{k} \pi\left(F_{i}\right) .
$$

Because the usual set union and set minus operation do not count the number of occurrences of an element, which is important in our paper, from now on we will consider any polytopes or sets as multisets which allow negative multiplicities. In other words, we consider any element of a multiset as a pair $(\mathbf{x}, m)$, where $m$ is the multiplicity of element $\mathbf{x}$. Then for any multisets $M_{1}, M_{2}$ and any integers $m, n$ and $i$, we define the following operators:

a) Scalar product: $i M_{1}=i \cdot M_{1}=\left\{(\mathbf{x}, i m) \mid(\mathbf{x}, m) \in M_{1}\right\}$.

b) Addition: $M_{1} \oplus M_{2}=\left\{(\mathbf{x}, m+n) \mid(\mathbf{x}, m) \in M_{1},(\mathbf{x}, n) \in M_{2}\right\}$.

c) Subtraction: $M_{1} \ominus M_{2}=M_{1} \oplus\left((-1) \cdot M_{2}\right)$.

It's clear that the following holds:

Lemma 2.3. For any polytope $P \subset \mathbb{R}^{d}, \forall R_{1}, \ldots, R_{k} \subset \mathbb{R}^{d-1}, \forall i_{1}, \ldots, i_{k} \in \mathbb{Z}$ :

$$
\rho^{+}\left(\bigoplus_{j=1}^{k} i_{j} R_{j}, P\right)=\bigoplus_{j=1}^{k} i_{j} \rho^{+}\left(R_{j}, P\right) .
$$

Definition 2.4. We say a set $S$ has weight $w$ if each of its elements has multiplicity either 0 or $w$. And $S$ is a signed set if it has weight 1 or -1 .

Let $P$ be a convex polytope. For any $y$ an interior point of $\pi(P)$, since $\pi$ is a continuous open map, the inverse image of $y$ contains an interior point of $P$. Thus $\pi^{-1}(y)$ intersects the boundary of $P$ exactly twice. For any $y$ a boundary point of $\pi(P)$, again because $\pi$ is an open map, we have that $\rho(y, P) \subset \partial P$, so $\rho(y, P)=\pi^{-1}(y) \cap \partial P$ is either one point or a line segment. We will only consider polytopes $P$ where $\rho(y, P)$ always has only one point for a boundary point $y$.

Lemma 2.5. If a polytope $P$ satisfies

$$
|\rho(y, P)|=1, \forall y \in \partial \pi(P),
$$

then $P$ has the following properties:

(i) For any $y \in I(\pi(P)), \pi^{-1}(y) \cap \partial P=\{p(y, P), n(y, P)\}$.

(ii) For any $y \in \partial \pi(P), \pi^{-1}(y) \cap \partial P=\rho(y, P)=p(y, P)=n(y, P)$, so $\rho^{+}(y, P)=\emptyset$.

(iii) Let $R$ be a region containing $I(\pi(P))$. Then

$$
\Omega(P)=\rho^{+}(R, P)=\bigoplus_{y \in R} \rho^{+}(y, P) .
$$

(iv) If $P=\bigsqcup_{i=1}^{k} P_{i}$, where the $P_{i}$ 's all satisfy (2.1), then $\Omega(P)=\bigoplus_{i=1}^{k} \Omega\left(P_{i}\right)$.

(v) The set of facets of $P$ are partitioned into the set of positive facets and the set of negative facets, i.e., there is no neutral facets.

(vi) $\pi$ gives a bijection between $P B(P) \cap N B(P)$ and $\partial \pi(P)$. 
The proof of this lemma is straightforward, so we won't include it here.

The main purpose of this paper is to discuss the number of lattice points in a polytope. Therefore, for simplicity, for any set $S \in \mathbb{R}^{d}$, we denote by $\mathcal{L}(S)=S \cap \mathbb{Z}^{d}$ the set of lattice points in $S$. It's not hard to see that $\mathcal{L}$ commutes with some of the operations we defined earlier, e.g. $\rho, \rho^{+}, \Omega$.

\section{LATTICE-FACE POLYTOPES}

A $d$-simplex is a polytope given as the convex hull of $d+1$ affinely independent points in $\mathbb{R}^{d}$.

Definition 3.1. We define lattice-face polytopes recursively. We call a 1-dimensional polytope a lattice-face polytope if it is integral.

For $d \geq 2$, we call a $d$-dimensional polytope $P$ with vertex set $V$ a lattice-face polytope if for any $d$-subset $U \subset V$,

a) $\pi(\operatorname{conv}(U))$ is a lattice-face polytope, and

b) $\pi\left(\mathcal{L}\left(H_{U}\right)\right)=\mathbb{Z}^{d-1}$, where $H_{U}$ is the affine space spanned by $U$. In other words, after dropping the last coordinate of the lattice of $H_{U}$, we get the $(d-1)$-dimensional lattice.

To understand the definition, let's look at examples of 2-polytopes.

Example 3.2. Let $P_{1}$ be the polytope with vertices $v_{1}=(0,0), v_{2}=(2,0)$ and $v_{3}=(2,1)$. Clearly, for any 2 -subset $U$, condition a) is always satisfied. When $U=\left\{v_{1}, v_{2}\right\}, H_{U}$ is $\{(x, 0) \mid x \in \mathbb{R}\}$. So $\pi\left(\mathcal{L}\left(H_{U}\right)\right)=\mathbb{Z}$, i.e., b) holds. When $U=$ $\left\{v_{1}, v_{3}\right\}, H_{U}$ is $\{(x, y) \mid x=2 y\}$. Then $\mathcal{L}\left(H_{U}\right)=\{(2 y, y) \mid y \in \mathbb{Z}\} \Rightarrow \pi\left(\mathcal{L}\left(H_{U}\right)\right)=$ $2 \mathbb{Z} \neq \mathbb{Z}$. When $U=\left\{v_{2}, v_{3}\right\}, H_{U}$ is $\{(2, y) \mid y \in \mathbb{R}\}$. Then $\pi\left(\mathcal{L}\left(H_{U}\right)\right)=\{2\} \neq \mathbb{Z}$. Therefore, $P_{1}$ is not a lattice-face polytope.

Let $P_{2}$ be the polytope with vertices $(0,0),(1,1)$ and $(2,0)$. One can check that $P_{2}$ is a lattice-face polytope.

The following lemma gives some properties of a lattice-face polytope.

Lemma 3.3. Let $P$ be a lattice-face d-polytope with vertex set $V$; then we have:

(i) $\pi(P)$ is a lattice-face $(d-1)$-polytope.

(ii) $m P$ is a lattice-face d-polytope, for any positive integer $m$.

(iii) $\pi$ induces a bijection between $\mathcal{L}(N B(P)$ ) (or $\mathcal{L}(P B(P))$ ) and $\mathcal{L}(\pi(P))$.

(iv) $\pi(\mathcal{L}(P))=\mathcal{L}(\pi(P))$.

(v) Any $d$-subset $U$ of $V$ forms a $(d-1)$-simplex. Thus $\pi(\operatorname{conv}(U))$ is a $(d-1)$ simplex.

(vi) Let $H$ be the affine space spanned by some $d$-subset of $V$. Then for any lattice point $y \in \mathbb{Z}^{d-1}$, we have that $\rho(y, H)$ is a lattice point.

(vii) $P$ is an integral polytope.

Proof. (i), (ii), (v) and (vi) can be checked directly from conditions a) and b) of the definition. (iii) and (iv) both follow from (vi). We prove (vii) by induction on $d$.

Any 1-dimensional lattice-face polytope is integral by definition.

For $d \geq 2$, suppose any $(d-1)$-dimensional lattice-face polytope is an integral polytope. Let $P$ be a $d$-dimensional lattice-face polytope with vertex set $V$. For any vertex $v_{0} \in V$, let $U$ be a subset of $V$ that contains $v_{0}$. Let $U=\left\{v_{0}, v_{1}, \ldots, v_{d-1}\right\}$. We know that $P^{\prime}=\pi(\operatorname{conv}(U))$ is a lattice-face $(d-1)$-simplex with vertices 
$\left\{\pi\left(v_{0}\right), \ldots, \pi\left(v_{d-1}\right)\right\}$. Thus, by the induction hypothesis, $P^{\prime}$ is an integral polytope. In particular, $\pi\left(v_{0}\right)$ is a lattice point. Therefore, $v_{0}=\rho\left(\pi\left(v_{0}\right), H_{U}\right)$ is a lattice point.

Remark 3.4. One sees that condition b) in the definition of lattice-face polytopes is equivalent to (vi).

The main theorem of this paper is to describe all of the coefficients of the Ehrhart polynomial of a lattice-face polytope.

Theorem 3.5. Let $P$ be a lattice-face d-polytope; then

$$
i(P, m)=\operatorname{Vol}(m P)+i(\pi(P), m)=\sum_{k=0}^{d} \operatorname{Vol}_{k}\left(\pi^{(d-k)}(P)\right) m^{k} .
$$

However, by Lemma 3.3/(ii),(iii), we have that

$$
i(P, m)=|\mathcal{L}(\Omega(m P))|+i(\pi(P), m) .
$$

Therefore, by Lemma 3.3/(i),(ii), to prove Theorem 3.5 it is sufficient to prove the following theorem:

Theorem 3.6. For any $P$ a lattice-face polytope,

$$
|\mathcal{L}(\Omega(P))|=\operatorname{Vol}(P) .
$$

Remark 3.7. We have an alternative definition of lattice-face polytopes, which is equivalent to Definition 3.1. Indeed, a $d$-polytope on a vertex set $V$ is a lattice-face polytope if and only if for all $k$ with $0 \leq k \leq d-1$,

$$
\text { for any }(k+1) \text {-subset } U \subset V, \pi^{d-k}\left(\mathcal{L}\left(H_{U}\right)\right)=\mathbb{Z}^{k},
$$

where $H_{U}$ is the affine space spanned by $U$. In other words, after dropping the last $d-k$ coordinates of the lattice of $H_{U}$, we get the $k$-dimensional lattice.

\section{A SIGNED DECOMPosition OF THE NONNEGATIVE PART OF A SIMPLEX IN GENERAL POSITION}

The volume of a polytope is not very hard to characterize. So our main problem is to find a way to describe the number of lattice points in the nonnegative part of a lattice-face polytope. We are going to do this via a signed decomposition.

4.1. Polytopes in general position. For the decomposition, we will work with a more general type of polytope (which contains the family of lattice-face polytopes).

Definition 4.1. We say that a $d$-polytope $P$ with vertex set $V$ is in general position if for any $k: 0 \leq k \leq d-1$, and any $(k+1)$-subset $U \subset V, \pi^{d-k}(\operatorname{conv}(U))$ is a $k$-simplex, where $\operatorname{conv}(U)$ is the convex hull of all of points in $U$.

By the alternative definition of lattice polytopes in Remark 3.7, it's easy to see that a lattice-face polytope is a polytope in general position. Therefore, the following discussion can be applied to lattice-face polytopes.

The following lemma states some properties of a polytope in general position. The proof is omitted.

Lemma 4.2. Given a d-polytope $P$ in general position with vertex set $V$, then

(i) $P$ satisfies (2.1).

(ii) $\pi(P)$ is a $(d-1)$-polytope in general position. 
(iii) For any nonempty subset $U$ of $V$, let $Q=\operatorname{conv}(U)$. If $U$ is has dimension $k(0 \leq k \leq d)$, then $\pi^{d-k}(Q)$ is a $k$-polytope in general position. In particular, for any facet $F$ of $P, \pi(F)$ is a $(d-1)$-polytope in general position.

(iv) For any triangulation of $P=\bigsqcup_{i=1}^{k} P_{i}$ without introducing new vertices, $\Omega(P)=\bigoplus_{i=1}^{k} \Omega\left(P_{i}\right)$. Thus, $\mathcal{L}(\Omega(P))=\bigoplus_{i=1}^{k} \mathcal{L}\left(\Omega\left(P_{i}\right)\right)$.

(v) If $F_{1}, F_{2}, \ldots, F_{\ell}$ are all the positive facets of $P$ and $F_{\ell+1}, \ldots, F_{k}$ are all the negative facets of $P$, then $\Omega(\pi(P))=\bigoplus_{i=1}^{\ell} \Omega\left(\pi\left(F_{i}\right)\right)=\bigoplus_{i=\ell+1}^{k} \Omega\left(\pi\left(F_{i}\right)\right)$.

(vi) For any hyperplane $H$ determined by one facet of $P$ and any $y \in \mathbb{R}^{d-1}$, $\rho(y, H)$ is one point.

(vii) For any $k: 0 \leq k \leq d-1$, any $(k+1)$-subset $U$ of $V$, and any $y_{1}, \ldots, y_{k} \in \mathbb{R}$, there exists a unique point $w \in \mathbb{R}^{d}$, such that the first $k$ coordinates of $w$ are $y_{1}, \ldots, y_{k}$ and $w$ is affinely dependent with the points in $U$.

Remark 4.3. By (iv), the problem of counting the number of lattice points in a polytope in general position is reduced to that of counting lattice points in a simplex in general position. In particular, together with the fact that $\operatorname{Vol}\left(\bigsqcup_{i=1}^{k} P_{i}\right)=$ $\sum_{i=1}^{k} \operatorname{Vol}\left(P_{i}\right)$, to prove Theorem 3.6 it is sufficient to prove the case when $P$ is a lattice-face simplex.

Therefore, we will only construct our decomposition in the case of simplices in general position. However, before the construction, we need one more proposition about the nonnegative part of a polytope in general position.

Proposition 4.4. Let $P$ be a d-polytope in general position with facets $F_{1}, F_{2}, \ldots$, $F_{k}$. Let $H$ be the hyperplane determined by $F_{k}$. For $i: 1 \leq i \leq k$, let $F_{i}^{\prime}=$ $\pi^{-1}\left(\pi\left(F_{i}\right)\right) \cap H$ and $Q_{i}=\operatorname{conv}\left(F_{i} \cup F_{i}^{\prime}\right)$. Then

$$
\Omega(P)=-\operatorname{sign}\left(F_{k}\right) \bigoplus_{i=1}^{k-1} \operatorname{sign}\left(F_{i}\right) \rho^{+}\left(\Omega\left(\pi\left(F_{i}\right)\right), Q_{i}\right) .
$$

Proof. We are going to just prove the case when $F_{k}$ is a negative facet; for the other case we can prove it analogously. Suppose $F_{1}, F_{2}, \ldots, F_{\ell}$ are positive facets and $F_{\ell+1}, \ldots, F_{k}$ are negative facets.

A special case of Lemma $2.5 /($ iii) is when $R=\Omega(\pi(P))$, so we have

$$
\Omega(P)=\rho^{+}(\Omega(\pi(P)), P)=\bigoplus_{y \in \Omega(\pi(P))} \rho^{+}(y, P) .
$$

Now for any points $a$ and $b$, we use $(a, b]$ to denote the half-open line segment between $a$ and $b$. Then, $\rho^{+}(y, P)=(n(y, P), p(y, P)]=(\rho(y, H), p(y, P)] \ominus$ $(\rho(y, H), n(y, P)]$. Therefore,

$$
\begin{aligned}
\Omega(P) & =\bigoplus_{y \in \Omega(\pi(P))}((\rho(y, H), p(y, P)] \ominus(\rho(y, H), n(y, P)]) \\
& =\left(\bigoplus_{y \in \Omega(\pi(P))}(\rho(y, H), p(y, P)]\right) \oplus\left(\bigoplus_{y \in \Omega(\pi(P))}(-1) \cdot(\rho(y, H), n(y, P)]\right) .
\end{aligned}
$$


By Lemma $4.2 /(\mathrm{v})$, we have $\Omega(\pi(P))=\bigoplus_{i=1}^{\ell} \Omega\left(\pi\left(F_{i}\right)\right)$. Therefore,

$$
\begin{aligned}
\bigoplus_{y \in \Omega(\pi(P))}(\rho(y, H), p(y, P)] & =\bigoplus_{i=1}^{\ell} \bigoplus_{y \in \Omega\left(\pi\left(F_{i}\right)\right)}(\rho(y, H), p(y, P)] \\
& =\bigoplus_{i=1}^{\ell} \bigoplus_{y \in \Omega\left(\pi\left(F_{i}\right)\right)}\left(\rho\left(y, F_{i}^{\prime}\right), \rho\left(y, F_{i}\right)\right] \\
& =\bigoplus_{i=1}^{\ell} \rho^{+}\left(\Omega\left(\pi\left(F_{i}\right)\right), Q_{i}\right) .
\end{aligned}
$$

Similarly, we will have

$$
\bigoplus_{y \in \Omega(\pi(P))}(-1) \cdot(\rho(y, H), n(y, P)]=\bigoplus_{i=\ell+1}^{k}(-1) \rho^{+}\left(\Omega\left(\pi\left(F_{i}\right)\right), Q_{i}\right) .
$$

Note that $\rho^{+}\left(\Omega\left(\pi\left(F_{k}\right)\right), Q_{k}\right)$ is the empty set. Thus, putting everything together, we get (4.1).

Now, we can use this proposition to inductively construct a decomposition of the nonnegative part $\Omega(P)$ of a $d$-simplex $P$ in general position into $d$ ! signed sets.

\section{Decomposition of $\Omega(P)$ :}

- If $d=1$, we do nothing: $\Omega(P)=\Omega(P)$.

- If $d \geq 2$, then by applying Proposition 4.4 to $P$ and letting $k=d+1$, we have

$$
\Omega(P)=-\operatorname{sign}\left(F_{d+1}\right) \bigoplus_{i=1}^{d} \operatorname{sign}\left(F_{i}\right) \rho^{+}\left(\Omega\left(\pi\left(F_{i}\right)\right), Q_{i}\right) .
$$

However, by Lemma $4.2 /(\mathrm{iii})$, each $\pi\left(F_{i}\right)$ is a $(d-1)$-simplex in general position. By the induction hypothesis, we have that $\Omega\left(\pi\left(F_{i}\right)\right)=$ $\bigoplus_{j=1}^{(d-1) !} S_{i, j}$, where $S_{i, j}$ 's are signed sets. Thus,

$$
\rho^{+}\left(\Omega\left(\pi\left(F_{i}\right)\right), Q_{i}\right)=\rho^{+}\left(\bigoplus_{j=1}^{(d-1) !} S_{i, j}, Q_{i}\right)=\bigoplus_{j=1}^{(d-1) !} \rho^{+}\left(S_{i, j}, Q_{i}\right) .
$$

Since each $\rho^{+}\left(S_{i, j}, Q_{i}\right)$ is a signed set, we have decomposed $\Omega(P)$ into $d$ ! signed sets.

Now we know that we can decompose $\Omega(P)$ into $d$ ! signed sets. But we still need to figure out what these sets are and which signs they have. In the next subsection, we are going to discuss the sign of a facet of a $d$-simplex, which is going to help us determine the signs in our decomposition.

4.2. The sign of a facet of a $d$-simplex. From now on, we will always use the following setup for a $d$-simplex unless otherwise stated:

Suppose $P$ is a $d$-simplex in general position with vertex set $V=\left\{v_{1}, v_{2}, \ldots, v_{d+1}\right\}$, where the coordinates of $v_{i}$ are $\mathbf{x}_{i}=\left(x_{i, 1}, x_{i, 2}, \ldots, x_{i, d}\right)$. 
For any $i$, we denote by $F_{i}$ the facet determined by vertices in $V \backslash\left\{v_{i}\right\}$ and $H_{i}$ the hyperplane determined by $F_{i}$.

For any $\sigma \in \mathfrak{S}_{d}$ and $k: 1 \leq k \leq d$, we define matrices $X_{V}(\sigma, k)$ and $Y_{V}(\sigma, k)$ to be the matrices

$$
\begin{gathered}
X_{V}(\sigma, k)=\left(\begin{array}{ccccc}
1 & x_{\sigma(1), 1} & x_{\sigma(1), 2} & \cdots & x_{\sigma(1), k} \\
1 & x_{\sigma(2), 1} & x_{\sigma(2), 2} & \cdots & x_{\sigma(2), k} \\
\vdots & \vdots & \vdots & \ddots & \vdots \\
1 & x_{\sigma(k), 1} & x_{\sigma(k), 2} & \cdots & x_{\sigma(k), k} \\
1 & x_{d+1,1} & x_{d+1,2} & \cdots & x_{d+1, k}
\end{array}\right)_{(k+1) \times(k+1)}, \\
Y_{V}(\sigma, k)=\left(\begin{array}{ccccc}
1 & x_{\sigma(1), 1} & x_{\sigma(1), 2} & \cdots & x_{\sigma(1), k-1} \\
1 & x_{\sigma(2), 1} & x_{\sigma(2), 2} & \cdots & x_{\sigma(2), k-1} \\
\vdots & \vdots & \vdots & \ddots & \vdots \\
1 & x_{\sigma(k), 1} & x_{\sigma(k), 2} & \cdots & x_{\sigma(k), k-1}
\end{array}\right)_{k \times k} .
\end{gathered}
$$

We also define $z_{V}(\sigma, k)$ to be

$$
z_{V}(\sigma, k)=\operatorname{det}\left(X_{V}(\sigma, k)\right) / \operatorname{det}\left(Y_{V}(\sigma, k)\right),
$$

where $\operatorname{det}(M)$ is the determinant of a matrix $M$.

We often omit the subscript $V$ for $X_{V}(\sigma, k), Y_{V}(\sigma, k)$ and $z_{V}(\sigma, k)$ if there is no confusion.

Now we can determine the sign of a facet $F_{i}$ of $P$ by looking at the determinants of these matrices, denoting by $\operatorname{sign}(x)$ the usual definition of sign of a real number $x$.

Lemma 4.5. We have

(i) $\forall i: 1 \leq i \leq d$ and $\forall \sigma \in \mathfrak{S}_{d}$ with $\sigma(d)=i$,

$$
\operatorname{sign}\left(F_{i}\right)=\operatorname{sign}(\operatorname{det}(X(\sigma, d)) / \operatorname{det}(X(\sigma, d-1))) .
$$

(ii) When $i=d+1$ and for $\forall \sigma \in \mathfrak{S}_{d}$,

$$
\operatorname{sign}\left(F_{d+1}\right)=-\operatorname{sign}(\operatorname{det}(X(\sigma, d)) / \operatorname{det}(Y(\sigma, d)))=-\operatorname{sign}(z(\sigma, d)) .
$$

Proof. For any $i: 1 \leq i \leq d+1$, let $v_{i}^{\prime}=\rho\left(\pi\left(v_{i}\right), H_{i}\right)$, i.e., $v_{i}^{\prime}$ is the unique point of the hyperplane spanned by $F_{i}$ which has the same coordinates as $v_{i}$ except for the last one. Suppose the coordinates of $v_{i}^{\prime}$ are $\left(x_{i, 1}, \ldots, x_{i, d-1}, x_{i, d}^{\prime}\right)$. Then $F_{i}$ is a positive facet if and only if $x_{i, d}<x_{i, d}^{\prime}$. Therefore,

$$
\operatorname{sign}\left(F_{i}\right)=-\operatorname{sign}\left(x_{i, d}-x_{i, d}^{\prime}\right) .
$$

$\forall i: 1 \leq i \leq d$ and $\forall \sigma \in \mathfrak{S}_{d}$ with $\sigma(d)=i$, because $v_{i}^{\prime}$ is in the hyperplane determined by $F_{i}$, we have that

$$
\operatorname{det}\left(\left(\begin{array}{ccccc}
1 & x_{\sigma(1), 1} & \cdots & x_{\sigma(1), d-1} & x_{\sigma(1), d} \\
\vdots & \vdots & \ddots & \vdots & \vdots \\
1 & x_{\sigma(d-1), 1} & \cdots & x_{\sigma(d-1), d-1} & x_{\sigma(d-1), d} \\
1 & x_{\sigma(d), 1} & \cdots & x_{\sigma(d), d-1} & x_{\sigma(d), d}^{\prime} \\
1 & x_{d+1,1} & \cdots & x_{d+1, d-1} & x_{d+1, d}
\end{array}\right)\right)=0 .
$$

Therefore,

$$
\operatorname{det}(X(\sigma, d))=(-1)^{2 d+1}\left(x_{i, d}-x_{i, d}^{\prime}\right) \operatorname{det}(X(\sigma, d-1))
$$


Thus,

$$
\operatorname{sign}(\operatorname{det}(X(\sigma, d)) / \operatorname{det}(X(\sigma, d-1)))=-\operatorname{sign}\left(x_{i, d}-x_{i, d}^{\prime}\right)=\operatorname{sign}\left(F_{i}\right) .
$$

We can similarly prove the formula for $i=d+1$.

4.3. Decomposition formulas. The following theorem describes the signed sets in our decomposition.

Theorem 4.6. Let $P$ be a d-simplex in general position with vertex set $V=$ $\left\{v_{1}, v_{2}, \ldots, v_{d+1}\right\}$, where the coordinates of $v_{i}$ are $\mathbf{x}_{i}=\left(x_{i, 1}, x_{i, 2}, \ldots, x_{i, d}\right)$. For any $\sigma \in \mathfrak{S}_{d}$, and $k: 0 \leq k \leq d-1$, let $v_{\sigma, k}$ be the point with the first $k$ coordinates the same as $v_{d+1}$ and affinely dependent with $v_{\sigma(1)}, v_{\sigma(2)}, \ldots, v_{\sigma(k)}, v_{\sigma(k+1)}$. (By Lemma 4.2/(vii), we know that there exists one and only one such point.) We also let $v_{\sigma, d}=v_{d+1}$. Then

$$
\Omega(P)=\bigoplus_{\sigma \in \mathfrak{S}_{d}} \operatorname{sign}(\sigma, P) S_{\sigma}
$$

where

$$
\operatorname{sign}(\sigma, P)=\operatorname{sign}(\operatorname{det}(X(\sigma, d))) \operatorname{sign}\left(\prod_{i=1}^{d} z(\sigma, i)\right)
$$

and

$$
S_{\sigma}=\left\{\mathbf{s} \in \mathbb{R}^{d} \mid \pi^{d-k}(\mathbf{s}) \in \Omega\left(\pi^{d-k}\left(\operatorname{conv}\left(\left\{v_{\sigma, 0}, \ldots, v_{\sigma, k}\right\}\right)\right)\right) \forall 1 \leq k \leq d\right\}
$$

is a set of weight 1 , i.e. a regular set.

Hence,

$$
\mathcal{L}(\Omega(P))=\bigoplus_{\sigma \in \mathfrak{S}_{d}} \operatorname{sign}(\sigma, P) \mathcal{L}\left(S_{\sigma}\right) .
$$

Proof. We prove it by induction on $d$.

When $d=1$, the only permutation $\sigma \in \mathfrak{S}_{1}$ is the identity permutation $\mathbf{1}$. One can check that $\operatorname{sign}(\mathbf{1}, P)=1$ and $S_{\mathbf{1}}=\Omega\left(\operatorname{conv}\left(v_{1}, v_{2}\right)\right)$. Thus (4.5) holds.

Assuming (4.5) holds for $d=d_{0} \geq 1$, we consider for $d=d_{0}+1$. For any $i: 1 \leq i \leq d, \pi\left(F_{i}\right)$ is a $(d-1)$-simplex in general position with vertex set $W=\left\{w_{1}, \ldots, w_{d}\right\}$, where $w_{j}=\left\{\begin{array}{ll}\pi\left(v_{j}\right), & j<i, \\ \pi\left(v_{j+1}\right), & j \geq i .\end{array}\right.$ Therefore, by the induction hypothesis,

$$
\Omega\left(\pi\left(F_{i}\right)\right)=\bigoplus_{\varsigma \in \mathfrak{S}_{d-1}} \operatorname{sign}\left(\varsigma, \pi\left(F_{i}\right)\right) S_{\varsigma}^{\prime}
$$

where

$$
\begin{gathered}
\operatorname{sign}\left(\varsigma, \pi\left(F_{i}\right)\right)=\operatorname{sign}\left(\operatorname{det}\left(X_{W}(\varsigma, d-1)\right)\right) \prod_{i=1}^{d-1} \operatorname{sign}\left(z_{W}(\varsigma, i)\right), \\
S_{\varsigma}^{\prime}=\left\{\mathbf{s} \in \mathbb{R}^{d-1} \mid \pi^{d-1-k}(\mathbf{s}) \in \Omega\left(\pi^{d-1-k}\left(\operatorname{conv}\left(\left\{w_{\varsigma, 0}, \ldots, w_{\varsigma, k}\right\}\right)\right)\right) \forall 1 \leq k \leq d-1\right\} . \\
\text { For any } \varsigma \in \mathfrak{S}_{d-1} \text {, if we let } \sigma \in \mathfrak{S}_{d} \text { with } \sigma(j)= \begin{cases}i, & j=d, \\
\varsigma(j), & \varsigma(j)<i, \text { then this } \\
\varsigma(j)+1, & \varsigma(j) \geq i,\end{cases}
\end{gathered}
$$
gives a bijection between $\varsigma \in \mathfrak{S}_{d-1}$ and $\sigma \in \mathfrak{S}_{d}$ with $\sigma(d)=i$. In particular, for 
any $j: 1 \leq j \leq d-1, w_{\varsigma(j)}=\pi\left(v_{\sigma(j)}\right)$. Hence,

$$
\operatorname{sign}\left(\varsigma, \pi\left(F_{i}\right)\right)=\operatorname{sign}(\operatorname{det}(X(\sigma, d-1))) \prod_{i=1}^{d-1} \operatorname{sign}(z(\sigma, i)) .
$$

Note that $w_{\varsigma, d-1}=w_{d}=\pi\left(v_{d+1}\right)=\pi\left(v_{\sigma, d-1}\right)$, so

$$
S_{\varsigma}^{\prime}=\left\{\mathbf{s} \in \mathbb{R}^{d-1} \mid \pi^{d-1-k}(\mathbf{s}) \in \Omega\left(\pi^{d-k}\left(\operatorname{conv}\left(\left\{v_{\sigma, 0}, \ldots, v_{\sigma, k}\right\}\right)\right)\right) \forall 1 \leq k \leq d-1\right\} .
$$

One can check that

$$
F_{i}^{\prime}=\pi^{-1}\left(\pi\left(F_{i}\right) \cap H_{d+1}=\operatorname{conv}\left(\left\{v_{1}, \ldots, v_{i-1}, v_{i+1}, \ldots, v_{d}, v_{\sigma, d-1}\right\}\right)\right.
$$

and $Q_{i}=\operatorname{conv}\left(F_{i} \cup F_{i}^{\prime}\right)=\operatorname{conv}\left(V \cup\left\{v_{\sigma, d-1}\right\} \backslash\left\{v_{i}\right\}\right)$. Hence,

$$
\rho^{+}\left(S_{\varsigma}^{\prime}, Q_{i}\right)=\left\{\mathbf{s} \in \mathbb{R}^{d} \mid \pi^{d-k}(\mathbf{s}) \in \Omega\left(\pi^{d-k}\left(\operatorname{conv}\left(\left\{v_{\sigma, 0}, \ldots, v_{\sigma, k}\right\}\right)\right)\right) \forall 1 \leq k \leq d\right\} .
$$

By letting $S_{\sigma}=\rho^{+}\left(S_{\varsigma}^{\prime}, Q_{i}\right)$ and $\operatorname{sign}(\sigma, P)=-\operatorname{sign}\left(F_{d+1}\right) \operatorname{sign}\left(F_{i}\right) \operatorname{sign}\left(\varsigma, \pi\left(F_{i}\right)\right)$ and using Lemma 4.5 , we get

$$
-\operatorname{sign}\left(F_{d+1}\right) \operatorname{sign}\left(F_{i}\right) \rho^{+}\left(\pi\left(F_{i}\right), Q_{i}\right)=\bigoplus_{\sigma \in \mathfrak{S}_{d}, \sigma(d)=i} \operatorname{sign}(\sigma, P) S_{\sigma} .
$$

Thus, together with (4.2), summing over all $i: 1 \leq i \leq d$ gives (4.5).

Corollary 4.7. If $P$ is a d-simplex in general position, then

$$
|\mathcal{L}(\Omega(P))|=\sum_{\sigma \in \mathfrak{S}_{d}} \operatorname{sign}(\sigma, P)\left|\mathcal{L}\left(S_{\sigma}\right)\right| .
$$

Therefore, if we can calculate the number of lattice points in $S_{\sigma}$, then we can calculate the number of lattice points in the nonnegative part of a $d$-simplex in general position. Although it's not so easy to find $\left|\mathcal{L}\left(S_{\sigma}\right)\right|$ for an arbitrary polytope, we can do it for any lattice-face $d$-polytope.

\section{LAtTice EnUmeration in $S_{\sigma}$ AND Bernoulli POlynomials}

In this section, we will count the number of lattice points in $S_{\sigma}$ when $P$ is a lattice-face $d$-simplex. This calculation involves Bernoulli polynomials.

5.1. Counting lattice points in $S_{\sigma}$. We say a map from $\mathbb{R}^{d} \rightarrow \mathbb{R}^{d}$ is lattice preserving if it is invertible and it maps lattice points to lattice points. Clearly, given a lattice preserving map $f$, for any set $S \in \mathbb{R}^{d}$ we have that $|\mathcal{L}(S)|=|\mathcal{L}(f(S))|$.

Let $P$ be a lattice face $d$-simplex with vertex set $V=\left\{v_{1}, \ldots, v_{d+1}\right\}$, where we use the same setup as before for $d$-simplices.

Given any $\sigma \in \mathfrak{S}_{d}$, recall that $S_{\sigma}$ is defined as in (4.7). To count the number of lattice points in $S_{\sigma}$, we want to find a lattice preserving affine transformation which simplifies the form of $S_{\sigma}$.

Before trying to find such a transformation, we will define more notation.

For any $\sigma \in \mathfrak{S}_{d}, k: 1 \leq k \leq d$ and $\mathbf{x}=\left(x_{1}, x_{2}, \ldots, x_{d}\right) \in \mathbb{R}^{d}$, we define the matrix $\tilde{X}(\sigma, k ; \mathbf{x})$ as

$$
\tilde{X}(\sigma, k ; \mathbf{x})=\left(\begin{array}{ccccc}
1 & x_{\sigma(1), 1} & x_{\sigma(1), 2} & \cdots & x_{\sigma(1), k} \\
1 & x_{\sigma(2), 1} & x_{\sigma(2), 2} & \cdots & x_{\sigma(2), k} \\
\vdots & \vdots & \vdots & \ddots & \vdots \\
1 & x_{\sigma(k), 1} & x_{\sigma(k), 2} & \cdots & x_{\sigma(k), k} \\
1 & x_{1} & x_{2} & \cdots & x_{k}
\end{array}\right)_{(k+1) \times(k+1)},
$$


and for $j: 0 \leq j \leq k$, let $\mathfrak{m}(\sigma, k ; j)$ be the minor of the matrix $\tilde{X}(\sigma, k ; \mathbf{x})$ obtained by omitting the last row and the $(j+1)$ th column. Then

$$
\operatorname{det}(\tilde{X}(\sigma, k ; \mathbf{x}))=(-1)^{k}\left(\mathfrak{m}(\sigma, k ; 0)+\sum_{j=1}^{k}(-1)^{j} \mathfrak{m}(\sigma, k ; j) x_{j}\right) .
$$

Note that $\mathfrak{m}(\sigma, k ; k)=\operatorname{det}(Y(\sigma, k))$. Therefore,

$$
\frac{\operatorname{det}(\tilde{X}(\sigma, k ; \mathbf{x}))}{\operatorname{det}(Y(\sigma, k))}=(-1)^{k} \frac{\mathfrak{m}(\sigma, k ; 0)}{\operatorname{det}(Y(\sigma, k))}+\sum_{j=1}^{k-1}(-1)^{k+j} \frac{\mathfrak{m}(\sigma, k ; j)}{\operatorname{det}(Y(\sigma, k))} x_{j}+x_{k}
$$

We will construct our transformation based on (5.2). Before that, we give the following lemma which discusses the coefficients in the right hand side of (5.2).

Lemma 5.1. Suppose $P$ is a lattice-face d-simplex. $\forall \sigma \in \mathfrak{S}_{d}, \forall k: 1 \leq k \leq d$, and $\forall j: 0 \leq j \leq k-1$, we have that

$$
\frac{\mathfrak{m}(\sigma, k ; j)}{\operatorname{det}(Y(\sigma, k))} \in \mathbb{Z}
$$

Proof. By the definition of lattice-face polytope and Lemma 3.3/(i), one can see that $\pi^{d-k}\left(\operatorname{conv}\left(v_{\sigma(1)}, \ldots, v_{\sigma(k)}, v_{d+1}\right)\right)=\operatorname{conv}\left(\pi^{d-k}\left(v_{\sigma(1)}\right), \ldots, \pi^{d-k}\left(v_{\sigma(k)}\right), \pi^{d-k}\left(v_{d+1}\right)\right)$ is a lattice-face $k$-polytope. Choose $U=\left\{\pi^{d-k}\left(v_{\sigma(1)}\right), \ldots, \pi^{d-k}\left(v_{\sigma(k)}\right)\right\}$; then $\pi\left(\mathcal{L}\left(H_{U}\right)\right)=\mathbb{Z}^{k-1}$, where $H_{U}$ is the affine space spanned by $U$. However,

$$
H_{U}=\left\{\mathbf{x}=\left(x_{1}, \ldots, x_{k}\right) \in \mathbb{R}^{k} \mid \operatorname{det}(\tilde{X}(\sigma, k ; \mathbf{x}))=0\right\} .
$$

Therefore, we must have that

$$
\operatorname{det}(\tilde{X}(\sigma, k ; \mathbf{x}))=0, \quad x_{1}, \ldots, x_{k-1} \in \mathbb{Z} \Rightarrow x_{k} \in Z .
$$

Let $x_{1}=\cdots=x_{k-1}=0$, then $\operatorname{det}(\tilde{X}(\sigma, k ; \mathbf{x}))=0$ implies that

$$
(-1)^{k} \frac{\mathfrak{m}(\sigma, k ; 0)}{\operatorname{det}(Y(\sigma, k))}+x_{k}=0 \Rightarrow \frac{\mathfrak{m}(\sigma, k ; 0)}{\operatorname{det}(Y(\sigma, k))}=(-1)^{k+1} x_{k} \in Z .
$$

For any $j: 1 \leq j \leq k-1$, let $x_{i}=x_{\sigma(j), i}+\delta_{i, j}$ for $1 \leq i \leq k-1$, where $\delta_{i, j}$ is the Kronecker delta function. Then, $\operatorname{det}(\tilde{X}(\sigma, k ; x))=0$ implies that

$$
\begin{aligned}
0= & (-1)^{k} \frac{\mathfrak{m}(\sigma, k ; 0)}{\operatorname{det}(Y(\sigma, k))}+\sum_{i=1}^{k-1}(-1)^{k+i} \frac{\mathfrak{m}(\sigma, k ; i)}{\operatorname{det}(Y(\sigma, k))} x_{\sigma(j), i} \\
& +x_{k}+(-1)^{k+j} \frac{\mathfrak{m}(\sigma, k ; j)}{\operatorname{det}(Y(\sigma, k))} \\
= & x_{k}-x_{\sigma(j), k}+(-1)^{k+j} \frac{\mathfrak{m}(\sigma, k ; j)}{\operatorname{det}(Y(\sigma, k))}
\end{aligned}
$$

where the second equality follows from the fact that $\left(x_{\sigma(j), 1}, \ldots, x_{\sigma(j), k}\right)$ is in $H_{U}$. Thus,

$$
\frac{\mathfrak{m}(\sigma, k ; j)}{\operatorname{det}(Y(\sigma, k))}=(-1)^{k+j+1}\left(x_{k}-x_{\sigma(j), k}\right) \in \mathbb{Z}
$$

Given this lemma, we have the following proposition. 
Proposition 5.2. There exists a lattice-preserving affine transformation $T_{\sigma}$ which maps $x=\left(x_{1}, x_{2}, \ldots, x_{d}\right) \in \mathbb{R}^{d}$ to

$$
\left(\frac{\operatorname{det}(\tilde{X}(\sigma, 1 ; \mathbf{x}))}{\operatorname{det}(Y(\sigma, 1))}, \frac{\operatorname{det}(\tilde{X}(\sigma, 2 ; \mathbf{x}))}{\operatorname{det}(Y(\sigma, 2))}, \ldots, \frac{\operatorname{det}(\tilde{X}(\sigma, d ; \mathbf{x}))}{\operatorname{det}(Y(\sigma, d))}\right) .
$$

Proof. Let $\alpha_{\sigma}=\left(-\frac{\mathfrak{m}(\sigma, 1 ; 0)}{\operatorname{det}(Y(\sigma, 1))}, \frac{\mathfrak{m}(\sigma, 2 ; 0)}{\operatorname{det}(Y(\sigma, 2))}, \ldots,(-1)^{d} \frac{\mathfrak{m}(\sigma, d ; 0)}{\operatorname{det}(Y(\sigma, d))}\right)$ and

$$
M_{\sigma}=\left(m_{\sigma, j, k}\right)_{d \times d},
$$

where

$$
m_{\sigma, j, k}= \begin{cases}1, & \text { if } j=k, \\ 0, & \text { if } j>k, \\ (-1)^{k+j} \frac{\mathfrak{m}(\sigma, k ; j)}{\operatorname{det}(Y(\sigma, k))}, & \text { if } j<k .\end{cases}
$$

We define $T_{\sigma}: \mathbb{R}^{d} \rightarrow \mathbb{R}^{d}$ by mapping $\mathbf{x}$ to $\alpha_{\sigma}+\mathbf{x} M_{\sigma}$. By (5.2),

$$
\alpha_{\sigma}+\mathbf{x} M_{\sigma}=\left(\frac{\operatorname{det}(\tilde{X}(\sigma, 1 ; \mathbf{x}))}{\operatorname{det}(Y(\sigma, 1))}, \frac{\operatorname{det}(\tilde{X}(\sigma, 2 ; \mathbf{x}))}{\operatorname{det}(Y(\sigma, 2))}, \ldots, \frac{\operatorname{det}(\tilde{X}(\sigma, d ; \mathbf{x}))}{\operatorname{det}(Y(\sigma, d))}\right) .
$$

Also, because all of the entries in $M_{\sigma}$ and $\alpha_{\sigma}$ are integers and the determinant of $M_{\sigma}$ is $1, T_{\sigma}$ is lattice preserving.

Corollary 5.3. Given $P$ a lattice-face polytope with vertex set $V=\left\{v_{1}, v_{2}, \ldots, v_{d+1}\right\}$, we have that

(i) $\forall i: 1 \leq i \leq d$, the last $d+1-i$ coordinates of $T_{\sigma}\left(v_{\sigma(i)}\right)$ are all zero.

(ii)

$$
T_{\sigma}\left(v_{d+1}\right)=(z(\sigma, 1), z(\sigma, 2), \ldots, z(\sigma, d)) .
$$

(iii) Recall that for $k: 0 \leq k \leq d-1, v_{\sigma, k}$ is the unique point with the first $k$ coordinates the same as $v_{d+1}$ and affinely dependent with $v_{\sigma(1)}, v_{\sigma(2)}, \ldots$, $v_{\sigma(k)}, v_{\sigma(k+1)}$. Then the first $k$ coordinates of $T_{\sigma}\left(v_{\sigma, k}\right)$ are the same as $T_{\sigma}\left(v_{d+1}\right)$ and the rest of the coordinates are zero. In other words,

$$
T_{\sigma}\left(v_{\sigma, k}\right)=(z(\sigma, 1), \ldots, z(\sigma, k), 0, \ldots, 0) .
$$

Proof. (i) This follows from that fact that $\operatorname{det}\left(\tilde{X}\left(\sigma, k ; \mathbf{x}_{\sigma(i)}\right)\right)=0$ if $1 \leq i \leq k \leq d$.

(ii) This follows from the fact that $\tilde{X}\left(\sigma, k ; \mathbf{x}_{d+1}\right)=X(\sigma, k)$ and $z(\sigma, k)=$ $\operatorname{det}(X(\sigma, k)) / \operatorname{det}(Y(\sigma, k))$.

(iii) Because for any $\mathbf{x} \in \mathbb{R}^{d}$, the $k$ th coordinate of $T_{\sigma}(\mathbf{x})$ only depends on the first $k$ coordinates of $\mathbf{x}, T_{\sigma}\left(v_{\sigma, k}\right)$ has the same first $k$ coordinates as $T_{\sigma}\left(v_{d+1}\right)$. $T_{\sigma}$ is an affine transformation. So $T_{\sigma}\left(v_{\sigma, k}\right)$ is affinely dependent with $T_{\sigma}\left(v_{\sigma(1)}\right)$, $T_{\sigma}\left(v_{\sigma(2)}\right), \ldots, T_{\sigma}\left(v_{\sigma(k)}\right), T_{\sigma}\left(v_{\sigma(k+1)}\right)$, the last $d-k$ coordinates of which are all zero. Therefore the last $d-k$ coordinates of $T_{\sigma}\left(v_{\sigma, k}\right)$ are all zero as well.

Recalling that $v_{\sigma, d}=v_{d+1}$, we are now able to describe $T_{\sigma}\left(S_{\sigma}\right)$.

Proposition 5.4. Let $\widehat{S}_{\sigma}=T_{\sigma}\left(S_{\sigma}\right)$. Then

$$
\mathbf{s}=\left(s_{1}, s_{2}, \ldots, s_{d}\right) \in \widehat{S}_{\sigma} \Leftrightarrow \forall 1 \leq k \leq d, s_{k} \in \Omega\left(\operatorname{conv}\left(0, \frac{z(\sigma, k)}{z(\sigma, k-1)} s_{k-1}\right)\right),
$$

where by convention we let $z(\sigma, 0)=1$ and $s_{0}=1$. 
Proof. $T_{\sigma}$ is an affine transformation whose corresponding matrix $M_{\sigma}$ is upper triangular. So $T_{\sigma}$ commutes with $\Omega, \pi$ and conv. Therefore,

$$
\widehat{S}_{\sigma}=\left\{\mathbf{s} \in \mathbb{R}^{d} \mid \pi^{d-k}(\mathbf{s}) \in \Omega\left(\pi^{d-k}\left(\operatorname{conv}\left(\left\{\widehat{v}_{\sigma, 0}, \ldots, \widehat{v}_{\sigma, k}\right\}\right)\right)\right) \forall 1 \leq k \leq d\right\},
$$

where $\widehat{v}_{\sigma, i}=T_{\sigma}\left(v_{\sigma, i}\right)=(z(\sigma, 1), \ldots, z(\sigma, i), 0, \ldots, 0)$, for $0 \leq i \leq d$.

(5.3) follows.

Because $T_{\sigma}$ is a lattice preserving map, $\left|\mathcal{L}\left(S_{\sigma}\right)\right|=\left|\mathcal{L}\left(\widehat{S}_{\sigma}\right)\right|$. Hence, our problem becomes to find the number of lattice points in $\widehat{S}_{\sigma}$. However, $\widehat{S}_{\sigma}$ is much nicer than $S_{\sigma}$. Actually, we can give a formula to calculate all of the sets having the same shape as $\widehat{S}_{\sigma}$.

Lemma 5.5. Given real nonzero numbers $b_{0}=1, b_{1}, b_{2}, \ldots, b_{d}$, let $a_{k}^{\prime}=b_{k} / b_{k-1}$ and $a_{k}=b_{k} /\left|b_{k+1}\right|, \forall k: 1 \leq k \leq d$. Let $S$ be the set defined by the following:

$$
\mathbf{s}=\left(s_{1}, s_{2}, \ldots, s_{d}\right) \in S \Leftrightarrow \forall 1 \leq k \leq d, s_{k} \in \Omega\left(\operatorname{conv}\left(0, a_{k}^{\prime} s_{k-1}\right)\right),
$$

where $s_{0}$ is set to 1 . Then

$$
|\mathcal{L}(S)|=\sum_{s_{1} \in \mathcal{L}\left(\Omega\left(\operatorname{conv}\left(0, a_{1}^{\prime}\right)\right)\right)} \sum_{s_{2} \in \mathcal{L}\left(\Omega\left(\operatorname{conv}\left(0, a_{2}^{\prime} s_{1}\right)\right)\right)} \cdots \sum_{s_{d} \in \mathcal{L}\left(\Omega\left(\operatorname{conv}\left(0, a_{d}^{\prime} s_{d-1}\right)\right)\right)} 1 .
$$

In particular, if $b_{d}>0$, then

$$
|\mathcal{L}(S)|=\sum_{s_{1}=1}^{\overline{\left\lfloor a_{1}\right\rfloor}} \sum_{s_{2}=1}^{\overline{\left\lfloor a_{2} s_{1}\right\rfloor}} \cdots \sum_{s_{d}=1}^{\overline{\left\lfloor a_{d} s_{d-1}\right\rfloor}} 1,
$$

where for any real number $x,\lfloor x\rfloor$ is the largest integer no greater than $x$ and $\bar{x}$ is defined as

$$
\bar{x}= \begin{cases}x, & \text { if } x \geq 0, \\ -x-1, & \text { if } x<0 .\end{cases}
$$

Note that $\overline{\lfloor x\rfloor} \in \mathbb{Z}_{\geq 0}$, and if any of the sums in (5.5) have upper bound equal to 0 , we consider the sum to be 0 .

Proof. (5.4) is straightforward. (5.5) follows from the facts that for any real numbers $x$,

$$
\mathcal{L}(\Omega(\operatorname{conv}(0, x)))= \begin{cases}\{z \in \mathbb{Z} \mid 1 \leq z \leq \overline{\lfloor x\rfloor}\}, & \text { if } x \geq 0, \\ \{z \in \mathbb{Z} \mid-\overline{\lfloor x\rfloor} \leq z \leq 0\}, & \text { if } x<0,\end{cases}
$$

the sign of $s_{i}$ is the same as the sign of $b_{i}$, and, because $b_{d}>0$, all the $s_{i}$ 's are nonzero.

We want to give a formula for the number of lattice points in $\widehat{S}_{\sigma}$ in the form of (5.5). We first need the condition " $b_{d}>0$ ", which in our case is that " $z(\sigma, d)>0$ ". However, for any $d$-simplex $P$ in general position, we can always find a way to order its vertices into $V=\left\{v_{1}, v_{2}, \ldots, v_{d+1}\right\}$, so that the corresponding $\operatorname{det}(X(\mathbf{1}, d))$ and $\operatorname{det}(Y(\mathbf{1}, d))$ are positive, where $\mathbf{1}$ stands for the identity permutation in $\mathfrak{S}_{d}$. Note $z(\sigma, d)$ is independent of $\sigma$. So it is positive.

Moreover, for lattice-polytopes, we have another good property of the $z(\sigma, k)$ 's which allows us to remove the \lfloor\rfloor operation in (5.5). 
Lemma 5.6. If $P$ is a lattice-polytope d-simplex, then

$$
z(\sigma, k) / z(\sigma, k-1) \in \mathbb{Z},
$$

where by convention $z(\sigma, 0)$ is set to 1 .

Proof. Let $P^{\prime}=T_{\sigma}(P)$ with vertex set $V^{\prime}=\left\{v_{1}^{\prime}, \ldots, v_{d+1}^{\prime}\right\}$, where $v_{i}^{\prime}=T_{\sigma}\left(v_{i}\right)$ with coordinates $\mathbf{x}_{i}^{\prime}=\left(x_{i, 1}^{\prime}, \ldots, x_{i, d}^{\prime}\right)$. Because $T_{\sigma}$ is an upper triangular lattice preserving map, $P^{\prime}$ is a lattice-face $d$-simplex as well. Similar to the proof of Lemma $5.1, \operatorname{conv}\left(\pi^{d-k}\left(v_{\sigma(1)}^{\prime}\right), \ldots, \pi^{d-k}\left(v_{\sigma(k)}^{\prime}\right), \pi^{d-k}\left(v_{d+1}^{\prime}\right)\right)$ is a lattice-face $k$-polytope. We choose $U=\left\{\pi^{d-k}\left(v_{\sigma(1)}^{\prime}\right), \ldots, \pi^{d-k}\left(v_{\sigma(k-1)}^{\prime}\right), \pi^{d-k}\left(v_{d+1}^{\prime}\right)\right\}$; then $\pi\left(\mathcal{L}\left(H_{U}\right)\right)=\mathbb{Z}^{k-1}$. Note that by Corollary 5.3/(i),(ii), we have that

a) the last 2 coordinates of $\pi^{d-k}\left(v_{\sigma(j)}^{\prime}\right)$ are both zero, for any $j: 1 \leq j \leq k-1$.

b) $\pi^{d-k}\left(v_{d+1}^{\prime}\right)=(z(\sigma, 1), \ldots, z(\sigma, k-1), z(\sigma, k))$.

Hence, $\left(x_{1}, \ldots, x_{k}\right) \in H_{U}$ if and only if $\operatorname{det}\left(\left(\begin{array}{cc}z(\sigma, k-1) & z(\sigma, k) \\ x_{k-1} & x_{k}\end{array}\right)\right)=0$, where we set $x_{0}=1$.

We have that for any $\left(x_{1}, \ldots, x_{k}\right) \in H_{U}$, if $x_{1}, \ldots, x_{k-1} \in \mathbb{Z}$, then $x_{k} \in \mathbb{Z}$. Thus, by setting $x_{k-1}=1$, we get $z(\sigma, k) / z(\sigma, k-1)=x_{k} \in \mathbb{Z}$.

Therefore, by Lemma 5.5 and Lemma 5.6, we have the following result.

Proposition 5.7. Let $P$ be a lattice-face $d$-simplex with vertex set $V$, where the order of vertices makes both $\operatorname{det}(X(\mathbf{1}, d))$ and $\operatorname{det}(Y(\mathbf{1}, d))$ positive. Define

$$
a(\sigma, k)=\frac{z(\sigma, k)}{|z(\sigma, k-1)|}, \forall k: 1 \leq k \leq d .
$$

Then

$$
\left|\mathcal{L}\left(S_{\sigma}\right)\right|=\sum_{s_{1}=1}^{\overline{a(\sigma, 1)}} \sum_{s_{2}=1}^{\overline{a(\sigma, 2) s_{1}}} \cdots \sum_{s_{d}=1}^{\overline{a(\sigma, d) s_{d-1}}} 1 .
$$

Because of (5.6), it's natural for us to define

$$
f_{d}\left(a_{1}, a_{2}, \ldots, a_{d}\right)=\sum_{s_{1}=1}^{a_{1}} \sum_{s_{2}=1}^{a_{2} s_{1}} \ldots \sum_{s_{d}=1}^{a_{d} s_{d-1}} 1
$$

for any positive integers $a_{1}, a_{2}, \ldots, a_{d}$.

Also, fixing $b_{0}=1$, we define

$$
g_{d}\left(b_{1}, b_{2}, \ldots, b_{d}\right)=f_{d}\left(b_{1} / b_{0}, b_{2} / b_{1}, \ldots, b_{d} / b_{d-1}\right),
$$

for any $\left(b_{1}, b_{2}, \ldots, b_{d}\right) \in\left(\mathbb{Z}_{>0}\right)^{d}$ such that $b_{i}$ is a multiple of $b_{i-1}(\forall 1 \leq i \leq d)$.

$f_{d}$ and $g_{d}$ are closely related to formula (5.6). In the next subsection, we will discuss Bernoulli polynomials and power sums, which are connected to $f_{d}$ and $g_{d}$, 
and then rewrite (5.6) in terms of $g_{d}$. Please refer to [3, Section 2.4] for other examples about Bernoulli polynomials and their relation to lattice polytopes.

5.2. Power sums and Bernoulli polynomials. The kth Bernoulli polynomial, $B_{k}(x)$, is defined as $[1$, p. 264]

$$
\frac{t e^{t x}}{e^{t}-1}=\sum_{k=0}^{\infty} B_{k}(x) \frac{t^{k}}{k !} .
$$

The Bernoulli polynomials satisfy [1, Theorem 12.19]

$$
B_{k}(1-x)=(-1)^{k} B_{k}(x), \forall k \geq 0,
$$

as well as the relation [1, Theorem 12.14]

$$
B_{k}(x+1)-B_{k}(x)=k x^{k-1}, \forall k \geq 1 .
$$

We call $B_{k}=B_{k}(0)$ a Bernoulli number. It satisfies [1, Theorem 12.16] that

$$
B_{k}(0)=0 \text {, for any odd number } k \geq 3 \text {. }
$$

For $k \geq 0$, let

$$
P_{k}(x)=\frac{B_{k+1}(x+1)-B_{k+1}}{k+1} .
$$

Given any $n$ a positive integer, by (5.10), we have that

$$
P_{k}(n)=\sum_{i=0}^{n} i^{k}= \begin{cases}\sum_{i=1}^{n} i^{k}, & \text { if } k \geq 1, \\ n+1, & \text { if } k=0\end{cases}
$$

Therefore, we call $P_{k}(x)$ the kth power sum polynomial. It's well known that for $k \geq 1$,

$$
\begin{aligned}
& P_{k}(x) \text { is a polynomial in } x \text { of degree } k+1, \\
& \text { the constant term of } P_{k}(x) \text { is } 0 \text {, i.e., } x \text { is a factor of } P_{k}(x) \text {, } \\
& \text { the leading coefficient of } P_{k}(x) \text { is } \frac{1}{k+1} .
\end{aligned}
$$

Lemma 5.8. For any $k \geq 1$,

$$
P_{k}(x)=(-1)^{k+1} P_{k}(-x-1) .
$$

Proof. It follows from (5.9) and (5.11).

Extension of the sum operation. Given $h=h(s)=\sum_{k \geq 0} h_{k} s^{k}$ a polynomial in $s$, the upper bound $u$ of a sum $\sum_{s=1}^{u} h(s)$ should be a positive integer in the usual definition. We extend this definition to allow $u$ (as well as the $h_{k}$ 's) to be in any polynomial ring over $\mathbb{R}$ using the formula

$$
\sum_{s=1}^{u} h(s):=h_{0} u+\sum_{k \geq 1} h_{k} P_{k}(u) .
$$

One can check that this extension agrees with the case when $u$ is a positive integer.

Since $f_{d}$ is defined by (5.7), which recursively uses the sum operation, we can use (5.16) to extend the domain of $f_{d}$ from $\mathbb{Z}_{>0}^{d}$ to $\mathbb{Z}^{d}$ or even $\mathbb{R}^{d}$. Hence, the domain of $g_{d}$ can be extended to $(\mathbb{R} \backslash\{0\})^{d}$. 
Lemma 5.9. $f_{d}\left(a_{1}, \ldots, a_{d}\right)$ is a polynomial in $a_{1}$ of degree $d$, and $\prod_{i=1}^{d} a_{i}$ is a factor of it. In particular, $f_{d}$ can be written as

$$
f_{d}\left(a_{1}, \ldots, a_{d}\right)=\sum_{k=1}^{d} f_{d, k}\left(a_{2}, \ldots, a_{d}\right) a_{1}^{k}
$$

where $f_{d, k}\left(a_{2}, \ldots, a_{d}\right)$ is a polynomial in $a_{2}, \ldots, a_{d}$ with a factor $\prod_{i=2}^{d} a_{i}$.

Proof. This can be proved by induction on $d$, using (5.12) and (5.13).

Lemma 5.10. Given $\left(a_{1}, a_{2}, \ldots, a_{d}\right) \in \mathbb{R}^{d}$,

$$
f_{d}\left(a_{1}, a_{2}, \ldots, a_{d}\right)=-\sum_{s_{1}=1}^{-a_{1}-1} \sum_{s_{2}=1}^{-a_{2} s_{1}} \sum_{s_{3}=1}^{a_{3} s_{2}} \cdots \sum_{s_{d}=1}^{a_{d} s_{d-1}} 1 .
$$

Proof.

$$
-\sum_{s_{1}=1}^{-a_{1}-1} \sum_{s_{2}=1}^{-a_{2} s_{1}} \sum_{s_{3}=1}^{a_{3} s_{2}} \cdots \sum_{s_{d}=1}^{a_{d} s_{d-1}} 1=-\sum_{s_{1}=1}^{-a_{1}-1} f_{d-1}\left(-a_{2} s_{1}, a_{3}, \ldots, a_{d}\right) .
$$

By (5.17) and (5.15), we have

$$
f_{d-1}\left(-a_{2} s_{1}, a_{3}, \ldots, a_{d}\right)=\sum_{k=1}^{d-1} f_{d-1, k}\left(a_{3}, \ldots, a_{d}\right)\left(-a_{2} s_{1}\right)^{k}
$$

and

$$
\sum_{s_{1}=1}^{-a_{1}-1} s_{1}^{k}=P_{k}\left(-a_{1}-1\right)=(-1)^{k+1} P_{k}\left(a_{1}\right)=(-1)^{k+1} \sum_{s_{1}=1}^{a_{1}} s_{1}^{k} .
$$

Therefore,

$$
\begin{aligned}
& -\sum_{s_{1}=1}^{-a_{1}-1} \sum_{s_{2}=1}^{-a_{2} s_{1}} \sum_{s_{3}=1}^{a_{3} s_{2}} \ldots \sum_{s_{d}=1}^{a_{d} s_{d-1}} 1=\sum_{s_{1}}^{a_{1}} \sum_{k=1}^{d-1} f_{d-1, k}\left(a_{3}, \ldots, a_{d}\right)\left(a_{2} s_{1}\right)^{k} \\
= & \sum_{s_{1}}^{a_{1}} f_{d-1}\left(a_{2} s_{1}, a_{3}, \ldots, a_{d}\right)=f_{d}\left(a_{1}, a_{2}, \ldots, a_{d}\right) .
\end{aligned}
$$

Proposition 5.11. Given $b_{0}=1, b_{1}, b_{2}, \ldots, b_{d} \in(\mathbb{R} \backslash\{0\})$ with $b_{d}>0$, let $a_{k}=$ $b_{k} /\left|b_{k-1}\right|$. Then

$$
g_{d}\left(b_{1}, b_{2}, \ldots, b_{d}\right)=f_{d}\left(b_{1}, \frac{b_{2}}{b_{1}}, \ldots, \frac{b_{d}}{b_{d-1}}\right)=\operatorname{sign}\left(\prod_{i=1}^{d} b_{i}\right) \sum_{s_{1}=1}^{\overline{a_{1}}} \sum_{s_{2}=1}^{\overline{a_{2} s_{1}}} \cdots \sum_{s_{d}=1}^{\overline{a_{d} s_{d}-1}} 1,
$$

where we always treat $s_{i}$ as positive when determining the meaning of $\overline{a_{i+1} s_{i}}$. That $i s$, for $a_{i+1}>0$, we set $\overline{a_{i+1} s_{i}}=a_{i+1} s_{i}$, and for $a_{i+1}<0$, we set $\overline{a_{i+1} s_{i}}=$ $-a_{i+1} s_{i}-1$. Note that this agrees with the original definition when the $a_{i}$ 's are all positive integers. 
Proof. We prove the proposition by induction on $d$. When $d=1$, it's trivial.

Assume (5.18) holds for $d=d_{0} \geq 1 . s_{1}$ is positive. Thus, by the induction hypothesis,

$$
\begin{aligned}
g_{d}\left(\frac{b_{2}}{\left|b_{1}\right|} s_{1}, \frac{b_{3}}{\left|b_{1}\right|} s_{1}, \ldots, \frac{b_{d}}{\left|b_{1}\right|} s_{1}\right) & =f_{d}\left(\frac{b_{2}}{\left|b_{1}\right|} s_{1}, \frac{b_{3}}{b_{2}}, \ldots, \frac{b_{d}}{b_{d-1}}\right) \\
& =\operatorname{sign}\left(\prod_{i=2}^{d} b_{i}\right) \sum_{s_{2}=1}^{\overline{a_{2} s_{1}}} \cdots \sum_{s_{d}=1}^{\overline{a_{d} s_{d-1}}} 1 .
\end{aligned}
$$

It's clear that (5.18) holds when $b_{1}>0$. In the case that $b_{1}<0,(5.18)$ follows from the above equation and Lemma 5.10.

Proposition 5.12. Let $P$ be a lattice-face $d$-simplex with vertex set $V$, where the order of vertices makes both $\operatorname{det}(X(\mathbf{1}, d))$ and $\operatorname{det}(Y(\mathbf{1}, d))$ positive. Then

$$
\left|\mathcal{L}\left(S_{\sigma}\right)\right|=\operatorname{sign}\left(\prod_{i=1}^{d} z(\sigma, i)\right) g_{d}(z(\sigma, 1), z(\sigma, 2), \ldots, z(\sigma, d)) .
$$

Therefore,

$$
|\mathcal{L}(\Omega(P))|=\sum_{\sigma \in \mathfrak{S}_{d}} \operatorname{sign}(\sigma) g_{d}(z(\sigma, 1), z(\sigma, 2), \ldots, z(\sigma, d))
$$

Proof. We can get (5.19) by comparing (5.6) and (5.18). Also, (5.20) follows from (4.9), (4.6), (5.19) and the fact that $\operatorname{det}(X(\sigma, d))=\operatorname{sign}(\sigma) \operatorname{det}(X(\mathbf{1}, d))$.

\section{Proof of the MAIN THEOREMS}

We now have all the ingredients but one to prove our main theorems: Theorem 3.5 and Theorem 3.6. The missing one is stated as the following proposition and will be proved in the next section.

Proposition 6.1. Let $V=\left\{v_{1}, v_{2}, \ldots, v_{d+1}\right\}$ be the vertex set of a d-simplex in general position, where the coordinates of $v_{i}$ are $\mathbf{x}_{i}=\left(x_{i, 1}, x_{i, 2}, \ldots, x_{i, d}\right)$. Recall that $X(\sigma, k), Y(\sigma, k)$ and $z(\sigma, k)$ are defined in $\S 4.2$ and $g_{d}$ is defined in (5.8). Then

$$
\sum_{\sigma \in \mathfrak{S}_{d}} \operatorname{sign}(\sigma) g_{d}(z(\sigma, 1), z(\sigma, 2), \ldots, z(\sigma, d))=\frac{1}{d !} \operatorname{det}(X(\mathbf{1}, d)),
$$

where 1 is the identity in $\mathfrak{S}_{d}$.

Given this proposition, we can prove the theorems.

Proof of Theorem 3.5 and Theorem 3.6. As we mentioned in Remark 4.3, to prove Theorem 3.6, it is sufficient to prove the case when $P$ is a lattice-face simplex.

When $P$ is a lattice-face $d$-simplex, we still assume that the order of the vertices of $P$ makes both $\operatorname{det}(X(\mathbf{1}, d))$ and $\operatorname{det}(Y(\mathbf{1}, d))$ positive. Thus, $(5.20),(6.1)$ and the fact that the volume of $P$ is $\frac{1}{d !}|\operatorname{det}(X(\mathbf{1}, d))|$ imply Theorem 3.6, and Theorem 3.5 follows.

Recall that we use $I(P)$ to denote the interior of a $d$-polytope $P$. We denote by $\widehat{i}(P, m)=\left|I(m P) \cap \mathbb{Z}^{d}\right|$ the number of lattice points in the interior of $m P$. 
Corollary 6.2. For any lattice-face d-polytope $P$, we have that

$$
\widehat{i}(P, m)=\operatorname{Vol}(m P)-\widehat{i}(\pi(P), m)=\sum_{k=0}^{d}(-1)^{d-k} \operatorname{Vol}_{k}\left(\pi^{(d-k)}(P)\right) m^{k} .
$$

Thus,

$$
i(P,-m)=(-1)^{d} \widehat{i}(P, m) .
$$

Proof. Since $P$ satisfies (2.1), by Lemma 2.5/(vi) and Lemma 3.3/(vi), $\pi$ induces a bijection between $\mathcal{L}(P B(P) \cap N B(P))$ and $\mathcal{L}(\partial \pi(P))$. Together with Lemma $3.3 /$ (ii), (iii), this implies

$$
\widehat{i}(P, m)=i(P, m)-i(\pi(P), m)-\widehat{i}(\pi(P), m) .
$$

Therefore, (6.2) and (6.3) follow from Theorem 3.5.

Note that (6.3) recovers the Ehrhart-Macdonald reciprocity law [6], which states that for any integral $d$-polytope, (6.3) holds.

The proof of Proposition 6.1 is self-contained and different from the rest of this paper. We put it separately in the next section.

\section{Proof of Proposition 6.1}

The purpose of this section is to prove Proposition 6.1 by showing both sides of (6.1) are equal to

$$
\frac{1}{d !} \sum_{\sigma \in \mathfrak{S}_{d}} \operatorname{sign}(\sigma) \prod_{j=1}^{d} z(\sigma, j)
$$

We always assume that $V=\left\{v_{1}, v_{2}, \ldots, v_{d+1}\right\}$ is the vertex set of a $d$-simplex in general position, where the coordinates of $v_{i}$ are $\mathbf{x}_{i}=\left(x_{i, 1}, x_{i, 2}, \ldots, x_{i, d}\right)$. We first give some new notation and definitions.

For all $k: 1 \leq k \leq d$, let $\widehat{\mathbf{x}}_{k}=\left(\widehat{x}_{k, 1}, \ldots, \widehat{x}_{k, d}\right)=\left(x_{k, 1}-x_{d+1,1}, \ldots, x_{k, d}-\right.$ $\left.x_{d+1, d}\right)=\mathbf{x}_{k}-\mathbf{x}_{d+1}$. Define

$$
\begin{gathered}
\widehat{X}_{V}(\sigma, k)=\left(\begin{array}{cccc}
\widehat{x}_{\sigma(1), 1} & \widehat{x}_{\sigma(1), 2} & \cdots & \widehat{x}_{\sigma(1), k} \\
\widehat{x}_{\sigma(2), 1} & \widehat{x}_{\sigma(2), 2} & \cdots & \widehat{x}_{\sigma(2), k} \\
\vdots & \vdots & \ddots & \vdots \\
\widehat{x}_{\sigma(k), 1} & \widehat{x}_{\sigma(k), 2} & \cdots & \widehat{x}_{\sigma(k), k}
\end{array}\right), \\
\widehat{Y}_{V}(\sigma, k)=\left(\begin{array}{cccc}
1 & \widehat{x}_{\sigma(1), 1} & \cdots & \widehat{x}_{\sigma(1), k-1} \\
1 & \widehat{x}_{\sigma(2), 1} & \cdots & \widehat{x}_{\sigma(2), k-1} \\
\vdots & \vdots & \ddots & \vdots \\
1 & \widehat{x}_{\sigma(k), 1} & \cdots & \widehat{x}_{\sigma(k), k-1}
\end{array}\right),
\end{gathered}
$$

and

$$
\widehat{z}_{V}(\sigma, k)=\operatorname{det}\left(\widehat{X}_{V}(\sigma, k)\right) / \operatorname{det}\left(\widehat{Y}_{V}(\sigma, k)\right) .
$$


Then

$$
\widehat{z}_{V}(\sigma, k)=(-1)^{k} z_{V}(\sigma, k)
$$

Again, when there is no confusion, we omit the subscript $V$ from $\widehat{X}_{V}(\sigma, k)$, $\widehat{Y}_{V}(\sigma, k)$ and $\widehat{z}_{V}(\sigma, k)$.

We define certain subsets of the symmetric group $\mathfrak{S}_{d}$, which we will use in our later proofs. We denote by $\mathfrak{S}_{T}$ the set of permutations on some set $T$ and use one-line notation for all permutations.

Definition 7.1. a) Let $(\Lambda, \Gamma, \Delta)$ be a partition of $[d]$ with the sizes of $\Lambda$ and $\Gamma$ to be $\ell$ and $i$, respectively. For any $\lambda \in \mathfrak{S}_{\Lambda}, \gamma \in S_{\Gamma}$ and $\delta \in \mathfrak{S}_{\Delta}$, we denote by $(\delta, \gamma, \lambda)$ the permutation $(\lambda(1), \ldots, \lambda(\ell), \gamma(1), \ldots, \gamma(i), \delta(1), \ldots$, $\delta(d-\ell-i))$. For fixed $\lambda$ and $\delta$, we denote by $\tilde{\mathfrak{S}}_{\lambda, d, \delta}$ the set of all of the permutations in the form of $(\lambda, \gamma, \delta)$.

b) In particular, when $\Delta$ is the empty set, i.e., $(\Lambda, \Gamma)$ is a partition of $[d]$, we simply write $\tilde{\mathfrak{S}}_{\lambda, d, \delta}$ as $\tilde{\mathfrak{S}}_{\lambda, d}$ which is the set of all of permutations in the form of $(\lambda, \gamma)$, for some fixed $\lambda \in S_{\Lambda}$.

c) We analogously define $\tilde{\mathfrak{S}}_{d, \delta}$ in the case that $\Lambda$ is the empty set, i.e., $(\Gamma, \Delta)$ is a partition of $[d]$.

7.1. Right side of (6.1). Because $\widehat{z}(\sigma, j)=(-1)^{j} z(\sigma, j)$ and

$$
\operatorname{det}(\widehat{X}(1, d))=(-1)^{d} \operatorname{det}(X(1, d)),
$$

to prove that the right side of (6.1) is equal to $\frac{1}{d !} \sum_{\sigma \in \mathfrak{S}_{d}} \operatorname{sign}(\sigma) \prod_{j=1}^{d} z(\sigma, j)$ is equivalent to showing that

$$
\sum_{\sigma \in \mathfrak{S}_{d}} \operatorname{sign}(\sigma) \prod_{j=1}^{d} \widehat{z}(\sigma, j)=(-1)^{\frac{d(d-1)}{2}} \operatorname{det}(\widehat{X}(\mathbf{1}, d)) .
$$

The following lemma gives a stronger statement. It involves $\tilde{\mathfrak{S}}_{d, \delta}$. For any $\sigma=$ $(\gamma, \delta) \in \tilde{\mathfrak{S}}_{d, \delta}$, and $\forall 1 \leq j \leq i$, $\operatorname{det}(\widehat{X}(\sigma, j))$ and $\operatorname{det}(\widehat{Y}(\sigma, j))$ do not depend on $\delta$. So we simply write them as $\operatorname{det}(\widehat{X}(\gamma, j))$ and $\operatorname{det}(\widehat{Y}(\gamma, j))$.

Lemma 7.2. For any $1 \leq i \leq d$, let $(\Gamma, \Delta)$ be a partition of $[d]$ with the size of $\Gamma$ equal to $i$. For any $\delta \in \mathfrak{S}_{\Delta}$ and $\gamma \in \mathfrak{S}_{\Gamma}$, we have that

$$
\sum_{\sigma \in \tilde{\mathfrak{S}}_{d, \delta}} \operatorname{sign}(\sigma) \prod_{j=1}^{i} \widehat{z}(\sigma, j)=(-1)^{\frac{i(i-1)}{2}} \operatorname{sign}((\gamma, \delta)) \operatorname{det}(\widehat{X}(\gamma, i)) .
$$

In particular, (7.2) holds.

Proof. We prove (7.3) by induction on $i$.

When $i=1$, there is only one $\sigma$ in $\tilde{\mathfrak{S}}_{d, \delta}$ and $\operatorname{sign}(\sigma)=\operatorname{sign}((\gamma, \delta))$. Together with the fact that $\operatorname{det}(\widehat{Y}(\gamma, 1))=1$, (7.3) holds.

Assuming that (7.3) holds when $i=i_{0} \geq 1$, consider $i=i_{0}+1$.

For any $m: 1 \leq m \leq i$, let $\Gamma^{(m)}=\Gamma \backslash\{\gamma(m)\}$ and $\Delta^{(m)}=\Delta \cup\{\gamma(m)\}$. Then $\left(\Gamma^{(m)}, \Delta^{(m)}\right)$ is a partition of $[d]$, where the size of $\Gamma^{(m)}$ is $i-1=i_{0}$. Let $\gamma^{(m)}=(\gamma(1), \ldots, \gamma(m-1), \gamma(m+1), \ldots, \gamma(i))$ and $\delta^{(m)}=(\gamma(m), \delta(1), \ldots, \delta(d-i))$. 
We know that $\operatorname{sign}\left(\left(\gamma^{(m)}, \delta^{(m)}\right)\right)=(-1)^{i+m} \operatorname{sign}((\gamma, \delta))$. Then by the induction hypothesis,

$$
\begin{aligned}
\sum_{\sigma \in \tilde{\mathfrak{S}}_{d, \delta}(m)} \operatorname{sign}(\sigma) \prod_{j=1}^{i-1} \widehat{z}(\sigma, j) & =(-1)^{\frac{(i-1)(i-2)}{2}} \operatorname{sign}\left(\left(\gamma^{(m)}, \delta^{(m)}\right)\right) \operatorname{det}\left(\widehat{X}\left(\gamma^{(m)}, i-1\right)\right) \\
& =(-1)^{\frac{(i-1)(i-2)}{2}+i+m} \operatorname{sign}((\gamma, \delta)) \operatorname{det}\left(\widehat{X}\left(\gamma^{(m)}, i-1\right)\right)
\end{aligned}
$$

However, $\left(\tilde{\mathfrak{S}}_{d, \delta(m)}\right)_{1 \leq m \leq i}$ gives a partition for $\tilde{\mathfrak{S}}_{d, \delta}$, and for any $\sigma \in \tilde{\mathfrak{S}}_{d, \delta}, \widehat{z}(\sigma, i)$ is an invariant. In particular, $\widehat{z}(\sigma, i)=\widehat{z}((\gamma, \delta), i)=\operatorname{det}(\widehat{X}(\gamma, i)) / \operatorname{det}(\widehat{Y}(\gamma, i))$. Therefore,

$$
\begin{aligned}
& \sum_{\sigma \in \tilde{\mathfrak{S}}_{d, \delta}} \operatorname{sign}(\sigma) \prod_{j=1}^{i} \widehat{z}(\sigma, j)=\sum_{m=1}^{i} \widehat{z}((\gamma, \delta), i) \sum_{\sigma \in \tilde{\mathfrak{S}}_{d, \delta}(j)} \operatorname{sign}(\sigma) \prod_{j=1}^{i-1} \widehat{z}(\sigma, j) \\
= & (-1)^{\frac{(i-1)(i-2)}{2}+i-1} \operatorname{sign}((\gamma, \delta)) \widehat{z}((\gamma, \delta), i) \sum_{m=1}^{i}(-1)^{m+1} \operatorname{det}\left(\widehat{X}\left(\gamma^{(m)}, i-1\right)\right) \\
= & (-1)^{\frac{i(i-1)}{2}} \operatorname{sign}((\gamma, \delta)) \widehat{z}((\gamma, \delta), i) \operatorname{det}(\widehat{Y}(\gamma, i)) \\
= & (-1)^{\frac{i(i-1)}{2}} \operatorname{sign}((\gamma, \delta)) \operatorname{det}(\widehat{X}(\gamma, i)) .
\end{aligned}
$$

Therefore, (7.3) holds. If we set $i=d$, then $\Delta=\emptyset$, and $\Gamma=[d]$. Letting $\gamma=\mathbf{1}$ be the identity in $\mathfrak{S}_{d}$, we obtain (7.2).

7.2. Left side of (6.1). The proof that

$$
\sum_{\sigma \in \mathfrak{S}_{d}} \operatorname{sign}(\sigma) g_{d}(z(\sigma, 1), \ldots, z(\sigma, d))=\frac{1}{d !} \sum_{\sigma \in \mathfrak{S}_{d}} \operatorname{sign}(\sigma) \prod_{j=1}^{d} z(\sigma, j)
$$

is relatively harder than what we did in the previous section. We need to use the following lemma.

Lemma 7.3. For any $0 \leq \ell+k \leq d-2$, given $p\left(y_{1}, \ldots, y_{\ell}\right)$ a function on $\ell$ variables, let $q(\sigma)=p(z(\sigma, 1), \ldots, z(\sigma, \ell)), \forall \sigma \in \mathfrak{S}_{d}$. Then

$$
\sum_{\sigma \in \mathfrak{S}_{d}} \operatorname{sign}(\sigma) q(\sigma) \frac{\prod_{j=\ell+1}^{d} z(\sigma, j)}{(z(\sigma, \ell+1))^{k+1}}=0 .
$$

Given this lemma, we are able to prove the following proposition which implies (7.4) when we set $\ell=0$.

Proposition 7.4. Define $s_{0}=1, z(\sigma, 0)=1$. For any $\ell: 0 \leq \ell \leq d$, we have that

$$
\begin{aligned}
& \sum_{\sigma \in \mathfrak{S}_{d}} \operatorname{sign}(\sigma) g_{d}(z(\sigma, 1), \ldots, z(\sigma, d)) \\
= & \frac{1}{(d-\ell) !} \sum_{\sigma \in \mathfrak{S}_{d}} \operatorname{sign}(\sigma) \sum_{s_{1}=1}^{\frac{z(\sigma, 1)}{z(\sigma, 0)} s_{0}} \cdots \sum_{s_{\ell}=1}^{\frac{z(\sigma, \ell)}{z(\sigma, \ell-1)}} s_{\ell-1} \frac{\prod_{j=\ell+1}^{d} z(\sigma, j)}{(z(\sigma, \ell))^{d-\ell}} s_{\ell}^{d-\ell} .
\end{aligned}
$$

Proof. We proceed by descending induction on $\ell$.

When $\ell=d,(7.6)$ holds by the definition of $g_{d}$.

When $\ell=d-1$, it's easy to check that (7.6) holds. 
Assuming (7.6) holds for $\ell=\ell_{0}+1 \leq d-1$, we consider $\ell=\ell_{0} \leq d-2$. By the induction hypothesis,

$$
\begin{aligned}
& \sum_{\sigma \in \mathfrak{S}_{d}} \operatorname{sign}(\sigma) g_{d}(z(\sigma, 1), \ldots, z(\sigma, d)) \\
= & \frac{1}{(d-\ell-1) !} \sum_{\sigma \in \mathfrak{S}_{d}} \operatorname{sign}(\sigma) \sum_{s_{1}=1}^{\frac{z(\sigma, 1)}{z(\sigma, 0)} s_{0}} \cdots \sum_{s_{\ell+1}=1}^{\frac{z(\sigma, \ell+1)}{z(\sigma, \ell)}} s_{\ell} \frac{\prod_{j=\ell+2}^{d} z(\sigma, j)}{(z(\sigma, \ell+1))^{d-\ell-1}} s_{\ell+1}^{d-\ell-1} \\
= & \frac{1}{(d-\ell-1) !} \sum_{\sigma \in \mathfrak{S}_{d}} \operatorname{sign}(\sigma) \sum_{s_{1}=1}^{\frac{z(\sigma, 1)}{z(\sigma, 0)} s_{0}} \cdots \sum_{s_{\ell}=1}^{\frac{z(\sigma, \ell)}{z(\sigma, \ell-1)}} s_{\ell-1} \frac{\prod_{j=\ell+2}^{d} z(\sigma, j)}{(z(\sigma, \ell+1))^{d-\ell-1}} \\
& \times P_{d-\ell-1}\left(\frac{z(\sigma, \ell+1)}{z(\sigma, \ell)} s_{\ell}\right) .
\end{aligned}
$$

Recall that $P_{d-\ell-1}(x)$ is the power sum polynomial. Note that $d-\ell-1 \geq 1$. By (5.12), (5.13) and (5.14), we can assume

$$
P_{d-\ell-1}(x)=\frac{1}{d-\ell} x^{d-\ell}+\sum_{m=1}^{d-\ell-1} c_{m} x^{m},
$$

where $c_{m} \in \mathbb{R}$.

For $\forall m: 1 \leq m \leq d-\ell-1$, defining $x_{0}=1$, let

$$
p_{m}\left(x_{1}, \ldots, x_{\ell}\right)=\sum_{s_{1}=1}^{\frac{x_{1}}{x_{0}} s_{0}} \cdots \sum_{s_{\ell}=1}^{\frac{x_{\ell}}{x_{\ell-1}} s_{\ell-1}}\left(\frac{s_{\ell}}{x_{\ell}}\right)^{m}
$$

Then $p_{m}$ is a function on $\ell$ variables. Let

$$
q_{m}(\sigma)=p_{m}(z(\sigma, 1), \ldots, z(\sigma, \ell))
$$

Then

$$
\begin{aligned}
& \sum_{\sigma \in \mathfrak{S}_{d}} \operatorname{sign}(\sigma) \sum_{s_{1}=1}^{\frac{z(\sigma, 1)}{z(\sigma, 0)} s_{0}} \cdots \sum_{s_{\ell}=1}^{\frac{z(\sigma, \ell)}{z(\sigma, \ell-1)} s_{\ell-1}} \frac{\prod_{j=\ell+2}^{d} z(\sigma, j)}{(z(\sigma, \ell+1))^{d-\ell-1}}\left(\frac{z(\sigma, \ell+1)}{z(\sigma, \ell)} s_{\ell}\right)^{m} \\
= & \sum_{\sigma \in \mathfrak{S}_{d}} \operatorname{sign}(\sigma) q_{m}(\sigma) \frac{\prod_{j=\ell+2}^{d} z(\sigma, j)}{(z(\sigma, \ell+1))^{d-\ell-1-m}} \\
= & \sum_{\sigma \in \mathfrak{S}_{d}} \operatorname{sign}(\sigma) q_{m}(\sigma) \frac{\prod_{j=\ell+1}^{d} z(\sigma, j)}{(z(\sigma, \ell+1))^{d-\ell-m}}=0 .
\end{aligned}
$$


The last equality is by (7.5). Therefore,

$$
\begin{aligned}
& \sum_{\sigma \in \mathfrak{S}_{d}} \operatorname{sign}(\sigma) g_{d}(z(\sigma, 1), \ldots, z(\sigma, d)) \\
= & \frac{1}{(d-\ell-1) !} \sum_{\sigma \in \mathfrak{S}_{d}} \operatorname{sign}(\sigma) \sum_{s_{1}=1}^{\frac{z(\sigma, 1)}{z(\sigma, 0)} s_{0}} \ldots \sum_{s_{\ell}=1}^{\frac{z(\sigma, \ell)}{z(\sigma, \ell-1)}} s_{\ell-1} \frac{\prod_{j=\ell+2}^{d} z(\sigma, j)}{(z(\sigma, \ell+1))^{d-\ell-1}} \\
& \times \frac{1}{d-\ell}\left(\frac{z(\sigma, \ell+1)}{z(\sigma, \ell)} s_{\ell}\right)^{d-\ell} \\
= & \frac{1}{(d-\ell) !} \sum_{\sigma \in \mathfrak{S}_{d}} \operatorname{sign}(\sigma) \sum_{s_{1}=1}^{\frac{z(\sigma, 1)}{z(\sigma, 0)}} s_{0} \\
& \frac{z(\sigma, \ell)}{z(\sigma, \ell-1)} s_{\ell-1}^{s_{\ell}=1} \frac{\prod_{j=\ell+1}^{d} z(\sigma, j)}{(z(\sigma, \ell))^{d-\ell}} s_{\ell}^{d-\ell} .
\end{aligned}
$$

Now we have everything we need to prove Proposition 6.1 .

Proof of Proposition 6.1. The proposition follows from (7.4), (7.2) and the facts that $\widehat{z}(\sigma, j)=(-1)^{j} z(\sigma, j)$ and $\operatorname{det}(\widehat{X}(1, d))=(-1)^{d} \operatorname{det}(X(1, d))$.

7.3. The proof of Lemma 7.3. It remains to prove Lemma 7.3, which is the most complicated part of this section. We will break the proof into several steps. The first lemma we need involves symmetric polynomials.

A symmetric polynomial on $d$ variables $y_{1}, \ldots, y_{d}$ is a polynomial that is unchanged by any permutation of its variables.

Lemma 7.5. For any $k \geq 0$, there exist symmetric polynomials $\phi_{i}^{k}\left(y_{1}, y_{2}, \ldots, y_{i}\right)$ on variables $y_{1}, y_{2}, \ldots, y_{i}$ for any $i: 1 \leq i \leq k+2$ and symmetric polynomials $\varphi_{j}^{k}\left(y_{1}, y_{2}, \ldots, y_{j}\right)$ on variables $y_{1}, y_{2}, \ldots, y_{j}$ for any $j: 2 \leq j \leq k+2$, so that

$$
\begin{gathered}
\phi_{1}^{k}=1, \quad \phi_{k+1}^{k}=1, \quad \phi_{k+2}^{k}=0, \\
\varphi_{2}^{k}\left(y_{1}, y_{2}\right)=\sum_{i=0}^{k} y_{1}^{i} y_{2}^{k-i}, \quad \varphi_{k+2}^{k}=1, \\
\forall 1 \leq i \leq k+1, \quad \phi_{i}^{k}\left(y_{1}, \ldots, y_{i}\right) y_{i+1}^{k+2-i}-\varphi_{i+1}^{k}\left(y_{1}, \ldots, y_{i+1}\right) y_{i+1} \\
=\quad-\phi_{i+1}^{k}\left(y_{1}, \ldots, y_{i+1}\right) y_{1} \cdots y_{i+1} .
\end{gathered}
$$

Proof. Proof by induction on $k$.

When $k=0, \phi_{1}^{0}=1, \phi_{2}^{0}=0, \varphi_{2}^{0}=1 \Rightarrow \phi_{1}^{0} y_{2}-\varphi_{2}^{0} y_{2}=-\phi_{2}^{0} y_{1} y_{2}$.

Assume that (7.7), (7.8) and (7.9) hold for $k=k_{0} \geq 0$. We check the case $k=k_{0}+1$.

We set

$$
\phi_{i}^{k}\left(y_{1}, \ldots, y_{i}\right)= \begin{cases}1, & \text { if } i=1 \\ \varphi_{i}^{k-1}\left(y_{1}, \ldots, y_{i}\right), & \text { if } 2 \leq i \leq k+1 \\ 0, & \text { if } i=k+2\end{cases}
$$

Note that $\phi_{k+1}^{k}=\varphi_{k+1}^{k-1}=1$ by the induction hypothesis. Thus, (7.7) holds. 
Now all of the $\phi_{i}^{k}$ 's are given. In order to satisfy (7.9), for $\forall 1 \leq i \leq k+1$, we set

$$
\varphi_{i+1}^{k}\left(y_{1}, \ldots, y_{i+1}\right)=\phi_{i}^{k}\left(y_{1}, \ldots, y_{i}\right) y_{i+1}^{k+1-i}+\phi_{i+1}^{k}\left(y_{1}, \ldots, y_{i+1}\right) y_{1} \cdots y_{i} \text {. }
$$

Hence, it is left to show that $\varphi_{i+1}^{k}$ 's are symmetric polynomials and satisfy (7.8).

When $i=1, \varphi_{2}^{k}\left(y_{1}, y_{2}\right)=\phi_{1}^{k}\left(y_{1}\right) y_{2}^{k}+\phi_{2}^{k}\left(y_{1}, y_{2}\right) y_{1}=y_{2}^{k}+\varphi_{2}^{k-1}\left(y_{1}, y_{2}\right) y_{1}=$ $y_{2}^{k}+\left(\sum_{i=0}^{k-1} y_{1}^{i} y_{2}^{k-1-i}\right) y_{1}=\sum_{i=0}^{k} y_{1}^{i} y_{2}^{k-i}$.

When $2 \leq i \leq k$, because the right hand side of (7.10) is symmetric in $y_{1}, y_{2}$, $\ldots, y_{i}$, it's enough to show that it is symmetric in $y_{1}$ and $y_{i+1}$. However,

$$
\begin{aligned}
\phi_{i}^{k}\left(y_{1}, \ldots, y_{i}\right) & =\varphi_{i}^{k-1}\left(y_{1}, \ldots, y_{i}\right) \\
& =\phi_{i-1}^{k-1}\left(y_{1}, \ldots, y_{i-1}\right) y_{i}^{k+1-i}+\phi_{i}^{k-1}\left(y_{1}, \ldots, y_{i}\right) y_{1} \cdots y_{i-1} .
\end{aligned}
$$

Because $\phi_{i}^{k}$ is symmetric, we can switch $y_{1}$ and $y_{i}$. So

$$
\phi_{i}^{k}\left(y_{1}, \ldots, y_{i}\right)=\phi_{i-1}^{k-1}\left(y_{2}, \ldots, y_{i}\right) y_{1}^{k+1-i}+\phi_{i}^{k-1}\left(y_{1}, \ldots, y_{i}\right) y_{2} \cdots y_{i} .
$$

Similarly,

$$
\phi_{i+1}^{k}\left(y_{1}, \ldots, y_{i+1}\right)=\phi_{i}^{k-1}\left(y_{2}, \ldots, y_{i+1}\right) y_{1}^{k-i}+\phi_{i+1}^{k-1}\left(y_{1}, \ldots, y_{i+1}\right) y_{2} \cdots y_{i+1} .
$$

Therefore,

$$
\begin{aligned}
& \varphi_{i+1}^{k}\left(y_{1}, \ldots, y_{i+1}\right) \\
= & \phi_{i-1}^{k-1}\left(y_{2}, \ldots, y_{i}\right) y_{1}^{k+1-i} y_{i+1}^{k+1-i}+\phi_{i}^{k-1}\left(y_{1}, \ldots, y_{i}\right) y_{2} \cdots y_{i} y_{i+1}^{k+1-i} \\
& +\phi_{i}^{k-1}\left(y_{2}, \ldots, y_{i+1}\right) y_{1}^{k+1-i} y_{2} \cdots y_{i}+\phi_{i+1}^{k-1}\left(y_{1}, \ldots, y_{i+1}\right) y_{1} y_{2}^{2} \cdots y_{i}^{2} y_{i+1}
\end{aligned}
$$

is symmetric in $y_{1}$ and $y_{i+1}$.

When $i=k+1, \varphi_{k+2}^{k}\left(y_{1}, \ldots, y_{i+1}\right)=\phi_{k+1}^{k}+\phi_{k+2}^{k} y_{1} \cdots y_{k+1}=1$.

Lemma 7.6. For any $0 \leq k \leq d-2,1 \leq i \leq k+2$, let $(\Gamma, \Delta)$ be a partition of $[d]$ with the size of $\Gamma$ equal to $i$. For any $\delta \in \mathfrak{S}_{\Delta}$ and $\gamma \in \mathfrak{S}_{\Gamma}$, we have that

$$
\begin{aligned}
& \sum_{\sigma \in \tilde{\mathfrak{S}}_{d, \delta}} \operatorname{sign}(\sigma) \frac{\prod_{j=1}^{i-1} \widehat{z}(\sigma, j)}{(\widehat{z}(\sigma, 1))^{k+1}} \\
= & (-1)^{\frac{i(i+1)}{2}-1} \operatorname{sign}((\gamma, \delta)) \frac{\phi_{i}^{k}\left(\widehat{x}_{\gamma(1), 1}, \ldots, \widehat{x}_{\gamma(i), 1}\right)}{\prod_{j=1}^{i}\left(\widehat{x}_{\gamma(j), 1}\right)^{k+2-i}} \operatorname{det}(\widehat{Y}(\gamma, i)) .
\end{aligned}
$$

Proof. We prove (7.11) by induction on $i$.

When $i=1$, there is only one $\sigma$ in $\tilde{\mathfrak{S}}_{d, \delta}$ and $\operatorname{sign}(\sigma)=\operatorname{sign}((\gamma, \delta))$. Together with the facts that $\phi_{1}^{k}=1, \widehat{z}(\sigma, 1)=\widehat{x}_{\sigma(1), 1}=\widehat{x}_{\gamma(1), 1}$ and $\operatorname{det}(\widehat{Y}(\gamma, 1))=1$, we conclude (7.11).

Assuming that (7.11) holds when $i=i_{0} \geq 1$, consider $i=i_{0}+1$.

For any $m: 1 \leq m \leq i$, let $\Gamma^{(m)}=\Gamma \backslash\{\gamma(m)\}$ and $\Delta^{(m)}=\Delta \cup\{\gamma(m)\}$. Then $\left(\Gamma^{(m)}, \Delta^{(m)}\right)$ is a partition of $[d]$, where the size of $\Gamma^{(m)}$ is $i-1=i_{0}$. Let $\gamma^{(m)}=(\gamma(1), \ldots, \gamma(m-1), \gamma(m+1), \ldots, \gamma(i))$ and $\delta^{(m)}=(\gamma(m), \delta(1), \ldots, \delta(d-i))$. 
Then by the induction hypothesis, we have that

$$
\begin{aligned}
& \sum_{\sigma \in \tilde{\mathfrak{S}}_{d, \delta(m)}} \operatorname{sign}(\sigma) \frac{\prod_{j=1}^{i-2} \widehat{z}(\sigma, j)}{(\widehat{z}(\sigma, 1))^{k+1}} \\
= & (-1)^{\frac{i(i-1)}{2}-1} \operatorname{sign}\left(\left(\gamma^{(m)}, \delta^{(m)}\right)\right) \\
& \times \frac{\phi_{i-1}^{k}\left(\widehat{x}_{\gamma^{(m)}(1), 1}, \ldots, \widehat{x}_{\gamma^{(m)}(i-1), 1}\right)}{\prod_{j=1}^{i-1}\left(\widehat{x}_{\gamma^{(m)}(j), 1}\right)^{k+3-i}} \operatorname{det}\left(\widehat{Y}\left(\gamma^{(m)}, i-1\right)\right) .
\end{aligned}
$$

However, $\left(\tilde{\mathfrak{S}}_{d, \delta}(m)\right)_{1 \leq m \leq i}$ gives a partition for $\tilde{\mathfrak{S}}_{d, \delta}$. Therefore,

$$
\sum_{\sigma \in \tilde{\mathfrak{S}}_{d, \delta}} \operatorname{sign}(\sigma) \frac{\prod_{j=1}^{i-1} \widehat{z}(\sigma, j)}{(\widehat{z}(\sigma, 1))^{k+1}}=\sum_{m=1}^{i} \sum_{\sigma \in \tilde{\mathfrak{S}}_{d, \delta}(j)} \operatorname{sign}(\sigma) \frac{\prod_{j=1}^{i-2} \widehat{z}(\sigma, j)}{(\widehat{z}(\sigma, 1))^{k+1}} \widehat{z}(\sigma, i-1) .
$$

But for any $\sigma \in \tilde{\mathfrak{S}}_{d, \delta^{(m)}}, \widehat{z}(\sigma, i-1)$ is an invariant. In particular, $\widehat{z}(\sigma, i-1)=$ $\widehat{z}\left(\left(\gamma^{(m)}, \delta^{(m)}\right), i-1\right)=\operatorname{det}\left(\widehat{X}\left(\gamma^{(m)}, i-1\right)\right) / \operatorname{det}\left(\widehat{Y}\left(\gamma^{(m)}, i-1\right)\right)$.

Hence,

$$
\begin{aligned}
& \sum_{\sigma \in \tilde{\mathfrak{S}}_{d, \delta}} \operatorname{sign}(\sigma) \frac{\prod_{j=1}^{i-1} \widehat{z}(\sigma, j)}{(\widehat{z}(\sigma, 1))^{k+1}} \\
= & \sum_{m=1}^{i}(-1)^{\frac{i(i-1)}{2}-1} \operatorname{sign}\left(\left(\gamma^{(m)}, \delta^{(m)}\right)\right) \\
& \times \frac{\phi_{i-1}^{k}\left(\widehat{x}_{\gamma^{(m)}(1), 1}, \ldots, \widehat{x}_{\gamma^{(m)}(i-1), 1}\right)}{\prod_{j=1}^{i-1}\left(\widehat{x}_{\gamma^{(m)}(j), 1}\right)^{k+3-i}} \operatorname{det}\left(\widehat{X}\left(\gamma^{(m)}, i-1\right)\right) .
\end{aligned}
$$

Note that

$$
\left(\widehat{x}_{\gamma(m), 1}\right)^{k+3-i} \prod_{j=1}^{i-1}\left(\widehat{x}_{\gamma^{(m)}(j), 1}\right)^{k+3-i}=\prod_{j=1}^{i}\left(\widehat{x}_{\gamma(j), 1}\right)^{k+3-i}
$$

and

$$
\operatorname{sign}\left(\left(\gamma^{(m)}, \delta^{(m)}\right)\right)=(-1)^{i+m} \operatorname{sign}((\gamma, \delta))
$$

Therefore,

$$
\begin{gathered}
\sum_{\sigma \in \tilde{\mathfrak{S}}_{d, \delta}} \operatorname{sign}(\sigma) \frac{\prod_{j=1}^{i-1} \widehat{z}(\sigma, j)}{(\widehat{z}(\sigma, 1))^{k+1}} \\
=(-1)^{\frac{i(i-1)}{2}-1+i} \frac{\operatorname{sign}((\gamma, \delta))}{\prod_{j=1}^{i}\left(\widehat{x}_{\gamma(j), 1}\right)^{k+3-i}} A,
\end{gathered}
$$

where

$$
\begin{aligned}
A & =\sum_{m=1}^{i}(-1)^{m} \phi_{i-1}^{k}\left(\widehat{x}_{\gamma^{(m)}(1), 1}, \ldots, \widehat{x}_{\gamma^{(m)}(i-1), 1}\right)\left(\widehat{x}_{\gamma(m), 1}\right)^{k+3-i} \operatorname{det}\left(\widehat{X}\left(\gamma^{(m)}, i-1\right)\right) \\
& =-\operatorname{det}\left(\left(\begin{array}{cccc}
\phi_{i-1}^{k}\left(\widehat{x}_{\gamma^{(1)}(1), 1}, \ldots, \widehat{x}_{\gamma^{(1)}(i-1), 1}\right)\left(\widehat{x}_{\gamma(1), 1}\right)^{k+3-i} & \widehat{x}_{\gamma(1), 1} & \ldots & \widehat{x}_{\gamma(1), i-1} \\
\phi_{i-1}^{k}\left(\widehat{x}_{\gamma^{(2)}(1), 1}, \ldots, \widehat{x}_{\gamma^{(2)}(i-1), 1}\right)\left(\widehat{x}_{\gamma(2), 1}\right)^{k+3-i} & \widehat{x}_{\gamma(2), 1} & \cdots & \widehat{x}_{\gamma(2), i-1} \\
\vdots & \vdots & \ddots & \vdots \\
\phi_{i-1}^{k}\left(\widehat{x}_{\gamma^{(i)}(1), 1}, \ldots, \widehat{x}_{\gamma^{(i)}(i-1), 1}\right)\left(\widehat{x}_{\gamma(i), 1}\right)^{k+3-i} & \widehat{x}_{\gamma(i), 1} & \cdots & \widehat{x}_{\gamma(i), i-1}
\end{array}\right)\right) .
\end{aligned}
$$


By (7.9), if we subtract the second column times $\varphi_{i}^{k}\left(\widehat{x}_{\gamma(1), 1}, \ldots, \widehat{x}_{\gamma(i), 1}\right)$ from the first column, then

$$
\begin{aligned}
A & =-\operatorname{det}\left(\left(\begin{array}{cccc}
-\phi_{i}^{k}\left(\widehat{x}_{\gamma(1), 1}, \ldots, \widehat{x}_{\gamma(i), 1}\right) \widehat{x}_{\gamma(1), 1} \cdots \widehat{x}_{\gamma(i), 1} & \widehat{x}_{\gamma(1), 1} & \cdots & \widehat{x}_{\gamma(1), i-1} \\
-\phi_{i}^{k}\left(\widehat{x}_{\gamma(1), 1}, \ldots, \widehat{x}_{\gamma(i), 1}\right) \widehat{x}_{\gamma(1), 1} \cdots \widehat{x}_{\gamma(i), 1} & \widehat{x}_{\gamma(2), 1} & \cdots & \widehat{x}_{\gamma(2), i-1} \\
\vdots & \vdots & \ddots & \vdots \\
-\phi_{i}^{k}\left(\widehat{x}_{\gamma(1), 1}, \ldots, \widehat{x}_{\gamma(i), 1}\right) \widehat{x}_{\gamma(1), 1} \cdots \widehat{x}_{\gamma(i), 1} & \widehat{x}_{\gamma(i), 1} & \cdots & \widehat{x}_{\gamma(i), i-1}
\end{array}\right)\right) \\
& =\phi_{i}^{k}\left(\widehat{x}_{\gamma(1), 1}, \ldots, \widehat{x}_{\gamma(i), 1}\right) \widehat{x}_{\gamma(1), 1} \cdots \widehat{x}_{\gamma(i), 1} \operatorname{det}(\widehat{Y}(\gamma, i)) .
\end{aligned}
$$

Therefore,

$$
\begin{gathered}
\sum_{\sigma \in \tilde{\mathfrak{S}}_{d, \delta}} \operatorname{sign}(\sigma) \frac{\prod_{j=1}^{i-1} \widehat{z}(\sigma, j)}{(\widehat{z}(\sigma, 1))^{k+1}} \\
=(-1)^{\frac{i(i+1)}{2}-1} \operatorname{sign}((\gamma, \delta)) \frac{\phi_{i}^{k}\left(\widehat{x}_{\gamma(1), 1}, \ldots, \widehat{x}_{\gamma(i), 1}\right)}{\prod_{j=1}^{i}\left(\widehat{x}_{\gamma(j), 1}\right)^{k+2-i}} \operatorname{det}(\widehat{Y}(\gamma, i)) .
\end{gathered}
$$

Corollary 7.7. For any $0 \leq \ell+k \leq d-2, i=k+2$, let $(\Lambda, \Gamma, \Delta)$ be a partition of $[d]$ with the sizes of $\lambda$ and $\Gamma$ equal to $\ell$ and $i$, respectively. For any $\delta \in \mathfrak{S}_{\Delta}$ and any $\lambda \in \mathfrak{S}_{\Lambda}$, we have that

$$
\sum_{\sigma \in \tilde{\mathfrak{S}}_{\lambda, d, \delta}} \operatorname{sign}(\sigma) \frac{\prod_{j=\ell+1}^{\ell+k+1} \widehat{z}(\sigma, j)}{(\widehat{z}(\sigma, \ell+1))^{k+1}}=0
$$

and

$$
\sum_{\sigma \in \tilde{\mathfrak{S}}_{\lambda, d, \delta}} \operatorname{sign}(\sigma) \frac{\prod_{j=\ell+1}^{\ell+k+1} z(\sigma, j)}{(z(\sigma, \ell+1))^{k+1}}=0
$$

Proof. Proof by induction on $\ell$.

When $\ell=0,(7.12)$ follows from Lemma 7.6 and the fact that $\phi_{k+2}^{k}=0$. Thus, (7.13) holds by (7.1).

We assume for $\ell=\ell_{0} \geq 0,(7.12)$ and (7.13) holds. We check the case $\ell=\ell_{0}+1$. Because (7.12) and (7.13) are equivalent by (7.1), it's enough to show (7.12).

Without loss of generality, we assume that $d \in \Lambda$ and $\lambda(1)=d$. For $1 \leq q \leq d-1$, define

$$
y_{p, q}= \begin{cases}\left(\widehat{x}_{p, q+1}-\widehat{x}_{d, q+1}\right) /\left(\widehat{x}_{p, 1}-\widehat{x}_{d, 1}\right), & \text { if } 1 \leq p \leq d-1, \\ \widehat{x}_{p, q+1} / \widehat{x}_{p, 1}, & \text { if } p=d\end{cases}
$$

Let $W$ be the vertex set $\left\{w_{1}, w_{2}, \ldots, w_{d-1}\right\}$, where the coordinates of $w_{p}$ are $\left(y_{p, 1}, y_{p, 2}, \ldots, y_{p, d-1}\right)$. For any $\sigma \in \tilde{\mathfrak{S}}_{\lambda, \delta}$, let $\varsigma=(\sigma(2), \sigma(3), \ldots, \sigma(d))$. Because $\lambda(1)=d, \varsigma \in \mathfrak{S}_{d-1}$. Clearly, $(\Lambda \backslash\{d\}, \Gamma, \Delta)$ is a partition for $[d-1]$ and $\varsigma \in \tilde{\mathfrak{S}}_{\lambda^{\prime}, d-1, \gamma}$, 
where $\lambda^{\prime}=(\lambda(2), \ldots, \lambda(\ell))$. Therefore, for $j \geq 2$,

$$
\begin{aligned}
& \operatorname{det}(\widehat{X}(\sigma, j)) \\
& =(-1)^{j-1} \operatorname{det}\left(\left(\begin{array}{cccc}
\widehat{x}_{\sigma(2), 1} & \widehat{x}_{\sigma(2), 2} & \cdots & \widehat{x}_{\sigma(2), j} \\
\vdots & \vdots & \ddots & \vdots \\
\widehat{x}_{\sigma(j), 1} & \widehat{x}_{\sigma(j), 2} & \cdots & \widehat{x}_{\sigma(j), j} \\
\widehat{x}_{d, 1} & \widehat{x}_{d, 2} & \cdots & \widehat{x}_{d, j}
\end{array}\right)\right) \\
& =(-1)^{j-1} \operatorname{det}\left(\left(\begin{array}{cccc}
\widehat{x}_{\sigma(2), 1}-\widehat{x}_{d, 1} & \widehat{x}_{\sigma(2), 2}-\widehat{x}_{d, 2} & \cdots & \widehat{x}_{\sigma(2), j}-\widehat{x}_{d, j} \\
\vdots & \vdots & \ddots & \vdots \\
\widehat{x}_{\sigma(j), 1}-\widehat{x}_{d, 1} & \widehat{x}_{\sigma(j), 2}-\widehat{x}_{d, 2} & \cdots & \widehat{x}_{\sigma(j), j}-\widehat{x}_{d, j} \\
\widehat{x}_{d, 1} & \widehat{x}_{d, 2} & \cdots & \widehat{x}_{d, j}
\end{array}\right)\right) \\
& =(-1)^{j-1} \widehat{x}_{d, 1} \prod_{p=2}^{j}\left(\widehat{x}_{\sigma(p), 1}-\widehat{x}_{d, 1}\right) \operatorname{det}\left(\left(\begin{array}{cccc}
1 & y_{\varsigma(1), 1} & \cdots & y_{\varsigma(1), j-1} \\
\vdots & \vdots & \ddots & \vdots \\
1 & y_{\varsigma(j-1), 1} & \cdots & y_{\varsigma(j-1), j-1} \\
1 & y_{d-1,2} & \cdots & y_{d-1, j-1}
\end{array}\right)\right) \\
& =(-1)^{j-1} \widehat{x}_{d, 1} \prod_{p=2}^{j}\left(\widehat{x}_{\sigma(p), 1}-\widehat{x}_{d, 1}\right) \operatorname{det}\left(X_{W}(\varsigma, j-1)\right) .
\end{aligned}
$$

Similarly,

$$
\operatorname{det}(\widehat{Y}(\sigma, j))=\prod_{p=2}^{j}\left(\widehat{x}_{\sigma(p), 1}-\widehat{x}_{d, 1}\right) \operatorname{det}\left(Y_{W}(\varsigma, j-1)\right) .
$$

Hence,

$$
\widehat{z}(\sigma, j)=\operatorname{det}(\widehat{X}(\sigma, j)) / \operatorname{det}(\widehat{Y}(\sigma, j))=(-1)^{j-1} \widehat{x}_{d, 1} z_{W}(\varsigma, j-1) .
$$

Note that $\operatorname{sign}(\sigma)=(-1)^{d-1} \operatorname{sign}(\varsigma)$. Hence, by the induction hypothesis,

$$
\begin{aligned}
& \sum_{\sigma \in \tilde{\mathfrak{S}}_{\lambda, d, \delta}} \operatorname{sign}(\sigma) \frac{\prod_{j=\ell+1}^{\ell+k+1} \widehat{z}(\sigma, j)}{(\widehat{z}(\sigma, \ell+1))^{k+1}} \\
= & \sum_{\varsigma \in \tilde{\mathfrak{S}}_{\lambda^{\prime}, d-1, \delta}}(-1)^{d-1} \operatorname{sign}(\varsigma) \frac{\prod_{j=\ell+1}^{\ell+k+1}(-1)^{j-1} z_{W}(\varsigma, j-1)}{\left(z_{W}(\varsigma, \ell)\right)^{k+1}}=0 .
\end{aligned}
$$

Proof of Lemma 7.3. Consider any partition $(\Lambda, \Gamma, \Delta)$ of $[d]$, where the size of $\Lambda$ is $\ell$ and the size of $\Gamma$ is $i=k+2$. If we fix $\lambda \in \mathfrak{S}_{\Lambda}$ and $\delta \in \mathfrak{S}_{\Delta}$, then $\forall \sigma \in \tilde{\mathfrak{S}}_{\lambda, d, \delta}$, $z(\sigma, j)$ is an invariant when $1 \leq j \leq \ell$ or $\ell+i \leq j \leq d$. Therefore, by (7.13),

$$
\sum_{\sigma \in \tilde{\mathfrak{S}}_{\lambda, d, \delta}} \operatorname{sign}(\sigma) q(\sigma) \frac{\prod_{j=\ell+1}^{d} z(\sigma, j)}{(z(\sigma, \ell+1))^{k+1}}=0 .
$$

But all of the $\tilde{\mathfrak{S}}_{\lambda, d, \delta}$ 's give a partition for $\mathfrak{S}_{d}$. Thus, (7.5) holds.

\section{EXAMPLES AND FURTHER DISCUSSION}

8.1. Examples of lattice-face polytopes. In this subsection, we use a fixed family of lattice-face polytopes to illustrate our results. Let $d=3$, and for any positive integer $k$, let $P_{k}$ be the polytope with the vertex set $V=\left\{v_{1}=(0,0,0), v_{2}=\right.$ 
$\left.(4,0,0), v_{3}=(3,6,0), v_{4}=(2,2,10 k)\right\}$. One can check that $P_{k}$ is a lattice-face polytope.

Example 8.1 (Example of Theorem 3.5). The volume of $P_{k}$ is $40 k$, and

$$
i\left(P_{k}, m\right)=40 k m^{3}+12 m^{2}+4 m+1 .
$$

$\pi\left(P_{k}\right)=\operatorname{conv}\{(0,0),(4,0),(3,6)\}$, where

$$
i\left(\pi\left(P_{k}\right), m\right)=12 m^{2}+4 m+1 .
$$

So

$$
i\left(P_{k}, m\right)=40 k m^{3}+i\left(\pi\left(P_{k}\right), m\right)
$$

which agrees with Theorem 3.5.

Example 8.2 (Example of formula (4.1)). $F_{4}=\operatorname{conv}\left(v_{1}, v_{2}, v_{3}\right)$ is a negative facet. The hyperplane determined by $F_{4}$ is $H=\left\{\left(x_{1}, x_{2}, x_{3}\right) \mid x_{3}=0\right\}$. Thus, $v_{4}^{\prime}=$ $\pi^{-1}\left(\pi\left(v_{4}\right)\right) \cap H=(2,2,0)$.

$F_{3}=\operatorname{conv}\left(v_{1}, v_{2}, v_{4}\right)$ is a positive facet. $\pi\left(F_{3}\right)=\operatorname{conv}((0,0),(4,0),(2,2))$. $\Omega\left(\pi\left(F_{3}\right)\right)=\pi\left(F_{3}\right) \backslash \operatorname{conv}((0,0),(4,0)) . F_{3}^{\prime}=\pi^{-1}\left(\pi\left(F_{3}\right)\right) \cap H=\operatorname{conv}\left(v_{1}, v_{2}, v_{4}^{\prime}\right)$. So

$$
\begin{gathered}
Q_{3}=\operatorname{conv}\left(F_{3} \cup F_{3}^{\prime}\right)=\operatorname{conv}\left(v_{1}, v_{2}, v_{4}, v_{4}^{\prime}\right), \\
\rho^{+}\left(\Omega\left(\pi\left(F_{3}\right)\right), Q_{3}\right)=Q_{3} \backslash F_{3}^{\prime} .
\end{gathered}
$$

$F_{2}=\operatorname{conv}\left(v_{1}, v_{3}, v_{4}\right)$ is a positive facet. $\pi\left(F_{2}\right)=\operatorname{conv}((0,0),(3,6),(2,2))$. $\Omega\left(\pi\left(F_{2}\right)\right)=\pi\left(F_{2}\right) \backslash(\operatorname{conv}((0,0),(2,2)) \cup \operatorname{conv}((2,2),(3,6))) . F_{2}^{\prime}=\pi^{-1}\left(\pi\left(F_{2}\right)\right) \cap H=$ $\operatorname{conv}\left(v_{1}, v_{3}, v_{4}^{\prime}\right)$. So

$$
\begin{gathered}
Q_{2}=\operatorname{conv}\left(F_{2} \cup F_{2}^{\prime}\right)=\operatorname{conv}\left(v_{1}, v_{3}, v_{4}, v_{4}^{\prime}\right), \\
\rho^{+}\left(\Omega\left(\pi\left(F_{2}\right)\right), Q_{2}\right)=Q_{2} \backslash\left(F_{2}^{\prime} \cup \operatorname{conv}\left(v_{1}, v_{4}, v_{4}^{\prime}\right) \cup \operatorname{conv}\left(v_{3}, v_{4}, v_{4}^{\prime}\right)\right) .
\end{gathered}
$$

$F_{1}=\operatorname{conv}\left(v_{2}, v_{3}, v_{4}\right)$ is a positive facet. $\pi\left(F_{1}\right)=\operatorname{conv}((4,0),(3,6),(2,2))$. $\Omega\left(\pi\left(F_{1}\right)\right)=\pi\left(F_{1}\right) \backslash \operatorname{conv}((4,0),(2,2)) . F_{1}^{\prime}=\pi^{-1}\left(\pi\left(F_{1}\right)\right) \cap H=\operatorname{conv}\left(v_{2}, v_{3}, v_{4}^{\prime}\right)$. So

$$
\begin{gathered}
Q_{1}=\operatorname{conv}\left(F_{1} \cup F_{1}^{\prime}\right)=\operatorname{conv}\left(v_{2}, v_{3}, v_{4}, v_{4}^{\prime}\right), \\
\rho^{+}\left(\Omega\left(\pi\left(F_{1}\right)\right), Q_{1}\right)=Q_{1} \backslash\left(F_{1}^{\prime} \cup \operatorname{conv}\left(v_{2}, v_{4}, v_{4}^{\prime}\right)\right) .
\end{gathered}
$$

Therefore,

$$
\Omega\left(P_{k}\right)=P_{k} \backslash F_{4}=-\operatorname{sign}\left(F_{4}\right) \bigoplus_{i=1}^{3} \operatorname{sign}\left(F_{i}\right) \rho^{+}\left(\Omega\left(\pi\left(F_{i}\right)\right), Q_{i}\right),
$$

which agrees with Proposition 4.4.

Example 8.3 (Example of decomposition). In this example, we decompose $P_{k}$ into 3 ! sets, where 5 of them have positive signs and one has negative sign, which is different from the cases for cyclic polytopes, where half of the sets have positive signs and the other half have negative signs.

Recall that $v_{\sigma, 3}=v_{4}=(2,2,10 k)$, for any $\sigma \in \mathfrak{S}_{3}$.

When $\sigma=123 \in \mathfrak{S}_{3}, v_{123,2}=v_{4}^{\prime}=(2,2,0), v_{123,1}=(2,0,0)$ and $v_{123,0}=v_{1}=$ $(0,0,0)$. Then

$$
S_{123}=\operatorname{conv}\left(\left\{v_{123, i}\right\}_{0 \leq i \leq 3}\right) \backslash \operatorname{conv}\left(\left\{v_{123, i}\right\}_{0 \leq i \leq 2}\right),
$$

with $\operatorname{sign}\left(123, P_{k}\right)=+1$. 
When $\sigma=213 \in \mathfrak{S}_{3}, v_{213,2}=v_{4}^{\prime}=(2,2,0), v_{213,1}=(2,0,0)$ and $v_{213,0}=v_{2}=$ $(4,0,0)$. Then

$$
S_{213}=\operatorname{conv}\left(\left\{v_{213, i}\right\}_{0 \leq i \leq 3}\right) \backslash\left(\operatorname{conv}\left(\left\{v_{213, i}\right\}_{0 \leq i \leq 2}\right) \cup \operatorname{conv}\left(\left\{v_{213, i}\right\}_{1 \leq i \leq 3}\right)\right),
$$

with $\operatorname{sign}\left(213, P_{k}\right)=+1$.

One can check that

$$
S_{123} \oplus S_{213}=\rho^{+}\left(\Omega\left(\pi\left(F_{3}\right)\right), Q_{3}\right) .
$$

When $\sigma=231 \in \mathfrak{S}_{3}, v_{231,2}=v_{4}^{\prime}=(2,2,0), v_{231,1}=(2,12,0)$ and $v_{231,0}=v_{2}=$ $(4,0,0)$. Then

$$
\begin{aligned}
S_{231}= & \operatorname{conv}\left(\left\{v_{231, i}\right\}_{0 \leq i \leq 3}\right) \backslash\left(\operatorname{conv}\left(\left\{v_{231, i}\right\}_{0 \leq i \leq 2}\right)\right. \\
& \cup \operatorname{conv}\left(\left\{v_{231, i}\right\}_{i=0,2,3} \cup \operatorname{conv}\left(\left\{v_{231, i}\right\}_{1 \leq i \leq 3}\right)\right),
\end{aligned}
$$

with $\operatorname{sign}\left(231, P_{k}\right)=+1$.

When $\sigma=321 \in \mathfrak{S}_{3}, v_{321,2}=v_{4}^{\prime}=(2,2,0), v_{321,1}=(2,12,0)$ and $v_{321,0}=v_{3}=$ $(3,6,0)$. Then

$$
\begin{aligned}
S_{321}= & \operatorname{conv}\left(\left\{v_{321, i}\right\}_{0 \leq i \leq 3}\right) \backslash\left(\operatorname{conv}\left(\left\{v_{321, i}\right\}_{0 \leq i \leq 2}\right)\right. \\
& \cup \operatorname{conv}\left(\left\{v_{321, i}\right\}_{i=0,2,3} \cup \operatorname{conv}\left(\left\{v_{321, i}\right\}_{1 \leq i \leq 3}\right)\right),
\end{aligned}
$$

with $\operatorname{sign}\left(321, P_{k}\right)=-1$.

One can check that

$$
S_{231} \ominus S_{321}=\rho^{+}\left(\Omega\left(\pi\left(F_{1}\right)\right), Q_{1}\right) .
$$

Similarly, we have that

$$
S_{132} \oplus S_{312}=\rho^{+}\left(\Omega\left(\pi\left(F_{2}\right)\right), Q_{2}\right) .
$$

Therefore, $\Omega\left(P_{k}\right)=\bigoplus_{\sigma \in \mathfrak{S}_{3}} \operatorname{sign}\left(\sigma, P_{k}\right) S_{\sigma}$, which coincides with Theorem 4.6.

8.2. Further discussion. Recall that Remark 3.7 gives an alternative definition for lattice-face polytopes. Note that in this definition, when $k=0$, satisfying (3.2) is equivalent to saying that $P$ is an integral polytope, which implies that the last coefficient of the Ehrhart polynomial of $P$ is 1 . Therefore, one may ask

Question 8.4. If $P$ is a polytope that satisfies (3.2) for all $k \in K$, where $K$ is a fixed subset of $\{0,1, \ldots, d-1\}$, can we say something about the Ehrhart polynomials of $P ?$

A special set $K$ can be chosen as the set of consecutive integers from 0 to $d^{\prime}$, where $d^{\prime}$ is an integer no greater than $d-1$. Based on some examples in this case, the Ehrhart polynomials seems to follow a certain pattern, so we conjecture the following:

Conjecture 8.5. Given $d^{\prime} \leq d-1$, if $P$ is a d-polytope with vertex set $V$ such that $\forall k: 0 \leq k \leq d^{\prime}$, (3.2) is satisfied, then for $0 \leq k \leq d^{\prime}$, the coefficient of $m^{k}$ in $i(P, m)$ is the same as in $i\left(\pi^{d-d^{\prime}}(P), m\right)$. In other words,

$$
i(P, m)=i\left(\pi^{d-d^{\prime}}(P), m\right)+\sum_{i=d^{\prime}+1}^{d} c_{i} m^{i} .
$$

When $d^{\prime}=0$, the condition on $P$ is simply that it is integral, and when $d^{\prime}=d-1$, we are in the case that $P$ is a lattice-face polytope. Therefore, for these two cases, this conjecture is true. 


\section{REFERENCES}

1. T. M. Apostol, Introduction to analytic number theory, Springer, 1976. MR0434929 (55:7892)

2. A. Barvinok, Lattice points, polyhedra, and complexity, Park City Math Institute Lecture Notes (Summer 2004), to appear.

3. M. Beck and S. Robins, Computing the continuous discretely: Integer-point enumeration in polyhedra, Springer (to appear). Preprint at http://math.sfsu.edu/beck/papers/ccd.html.

4. E. Ehrhart, Sur les polyèdres rationnels homothétiques à $n$ dimensions, C. R. Acad. Sci. Paris 254 (1962), 616-618. MR0130860 (24:A714)

5. F. Liu, Ehrhart polynomials of cyclic polytopes, Journal of Combinatorial Theory Ser. A 111 (2005), 111-127. MR2144858 (2006a:05012)

6. I. G. Macdonald, Polynomials associated with finite cell-complexes, J. London Math. Soc. 2 (1971), 4:181-192. MR0298542 (45:7594)

Department of Mathematics, Room 2-333, 77 Massachusetts Avenue, Massachusetts Institute of Technology, Cambridge, Massachusetts 02139

E-mail address: fuliu@math.mit.edu

Current address: Department of Mathematics, University of California, Davis, One Shields Avenue, Davis, California 95616

E-mail address: fuliu@math.ucdavis.edu 